\title{
SURFACE-MODIFIED COALS FOR ENHANCED CATALYST DISPERSION AND LIQUEFACTION
}

FINAL REPORT

September 1, 1996 to August 31, 1999

Dr. Yaw D. Yeboah

Department of Engineering

Clark Atlanta University

Atlanta, Georgia 30314

\section{PREPARED FOR THE UNITED STATES DEPARTMENT OF ENERGY} UNDER CONTRACT NUMBER DE-FG22-95PC95229--07 


\section{TABLE OF CONTENTS}

Description

Page

SUMMARY

4

1.0 INTRODUCTION

6

2.0 PROJECT OBJECTIVES

8

$\begin{array}{lll}2.1 & \text { Research objectives } & 8\end{array}$

2.2 Educational Objectives 10

$\begin{array}{lll}2.3 & \text { Impact of the proposed research } & 11\end{array}$

3.0 SCIENTIFIC BACKGROUND 12

3.1 Coal Structure and Properties 12

3.2 Coal Surface Chemistry 14

3.3 Coal Liquefaction History 17

3.4 Coal Liquefaction Developments 19

3.5 Catalysts and Coal Liquefaction 20

3.6 Catalyst Addition to Coal 21

$\begin{array}{lll}3.7 & \text { Coal and Surfactants } & 25\end{array}$

$\begin{array}{lll}4.0 & \text { TECHNICAL APPROCAH } & 27\end{array}$

$\begin{array}{lll}4.1 & \text { Materials } & 27\end{array}$

4.1.1 Description of the Coal 27

$\begin{array}{lll}\text { 4.1.2 Reagents and Chemicals } & 28\end{array}$

4.2 Preparation of the Coal Water Slurries 30

4.3 Zeta Potential Measurements 30

4.4 Surface Area Analysis 31

4.5 FTIR of Coal Samples 33

4.6 Atomic Force Microscopy 34

4.7 Luminescence Intensity Measurements 35

4.8 UV-Vis Measurements 35 
Desciption $\quad$ Page

4.9 Liquefaction Studies 36

5.0 RESULTS AND DISCUSSION 37

5.1 Zeta Potential Measurements 37

5.2 Surface Area Analysis 40

5.3 Molybdenum Adsorption of Surfactant Treated Coal vs. pH 43

5.4 FTIR of Coal Samples 46

5.5 Atomic Force Microscopy 47

5.6 Luminescence Intensity Measurements 49

5.7 Liquefaction Results $\quad 52$

5.8 Qualitative Analysis of Surfactant Adsorption by UV Measurements 54

6.0 OTHER PROGRAM ACCOMPLISHMENTS 55

6.1 Student Supported 55

6.2 Student Thesis Produced 55

6.3 Publications and Presentations 56

$\begin{array}{lll}6.3 & \text { Special Awards } & 56\end{array}$

$\begin{array}{lll}7.0 & \text { CONCLUSIONS } & 56\end{array}$

$\begin{array}{lll}8.0 & \text { RECOMMENDATIONS } & 57\end{array}$

$\begin{array}{lll}9.0 & \text { ACKNOWLEDGMENTS } & 58\end{array}$

$\begin{array}{lll}10.0 & \text { REFERENCES } & 58\end{array}$ 


\section{SUMMARY}

This is the final report of the Department of Energy Sponsored project DE-FGF2295PC95229 entitled, surface modified coals for enhanced catalyst dispersion and liquefaction. The aims of the study were to enhance catalyst loading and dispersion in coal for improved liquefaction by preadsorption of surfactants and catalysts on the coal and to train and educate minority scientists in catalysts and separation science.

Illinois No. 6 Coal (DEC-24) was selected for the study. The surfactants investigated included dodecyl dimethyl ethyl ammonium bromide (DDAB), a cationic surfactant, sodium dodecyl sulfate, an anionic surfactant, and Triton $\mathrm{x}-100$, a neutral surfactant. Ammonium molybdate tetrahydrate was used as the molybdenum catalyst precursor. Zeta potential, BET, FTIR, AFM, UV-Vis and luminescience intensity measurements were undertaken to assess the surface properties and the liquefaction activities of the coal.

The parent coal had a net negative surface charge over the $\mathrm{pH}$ range 2-12. However, in the presence of DDAB the negativity of the surface charge decreased. At higher concentrations of DDAB, a positive surface charge resulted. In contrast to the effect of $\mathrm{DDAB}$, the zeta potential of the coal became more negative than the parent coal in the presence of SDS. Adsorption of Triton reduced the net negative charge density of the coal samples.

The measured surface area of the coal surface was about $30 \mathrm{~m}^{2} / \mathrm{g}$ compared to $77 \mathrm{~m}^{2} / \mathrm{g}$ after being washed with deionized water. Addition of the surfactants decreased the surface area of the samples. Adsorption of the molybdenum catalyst increased the surface area of the coal sample. The adsorption of molybdenum on the coal was significantly promoted by preadsorption of DDAB and SDS. Molybdenum adsorption showed that, over a wide range of concentrations and $\mathrm{pH}$ values, the DDAB treated coal adsorbed a higher amount of molybdenum than the samples treated with SDS. The 
infrared spectroscopy (FTIR) and the atomic force microscopy (AFM) also provided evidence that confirmed the adsorption of the surfactants onto the coal surface. The luminescence measurements showed that the coal and solid surfactants luminescence weakly. No statistically significant influence was observed that resulted from the action of the surfactants or surfactant-molybdenum catalyst.

Interestingly, the liquefaction results produced data that indicated the use of surfactants did not significantly improve the liquefaction activity of the coal as had initially been hypothesized. The UV-adsorption tests provided evidence that suggest that this may have been due to oversaturation. Detailed discussions of the results and recommendations for future work are provided. 


\subsection{INTRODUCTION}

The expeditious consumption of the earth's oil supply is quickly exceeding the rate at which scientists have found a replacement for this valuable natural resource. This has stimulated a growing field of research dedicated to finding alternative fuel sources. Coal reserves represent the largest of the world's energy resources (13.6 x $10^{12}$ tons). As evidenced in Figures 1 and 2, U.S.A., U.S.S.R., and the People's Republic of China control over $80 \%$ of these reserves. The United States possesses a vast amount of coal resources (397.7 x $10^{9}$ tons), which exist as one of the many possible alternative energy sources[1].

Advanced technologies for coal liquefaction and gasification can significantly increase coal utilization. The application of the conventional hydrating catalysts to coal liquefaction and upgrading of the resulting liquids to produce fuels of environmentally acceptable quality has not been particularly successful, a situation which has stimulated research into alternative catalysts. The poor performance of the conventional catalysts is principally due to differences in the structure and composition of coal and petroleum. Since the former is more aromatic in nature, it is more susceptible to retrogressive cokeforming side reactions during thermal processing and is therefore more prone to cause catalyst deactivation. This problem is augmented by deposition of inherent inorganic materials on the catalyst, the encapsulation of catalyst active sites by chemisorbed molecules [2], and the lack of appropriate technologies for catalyst recovery and recycle [3].

Since the development of catalytic coal liquefaction was initiated in 1924 [2], a wide variety of supported and unsupported catalysts have been applied [4-18]. Excellent review articles on the subject are available [2,4,9-11]. The catalysts consist of metal 
oxides, metal sulfides, metal halides, molten metals, and metal complexes. Catalysts

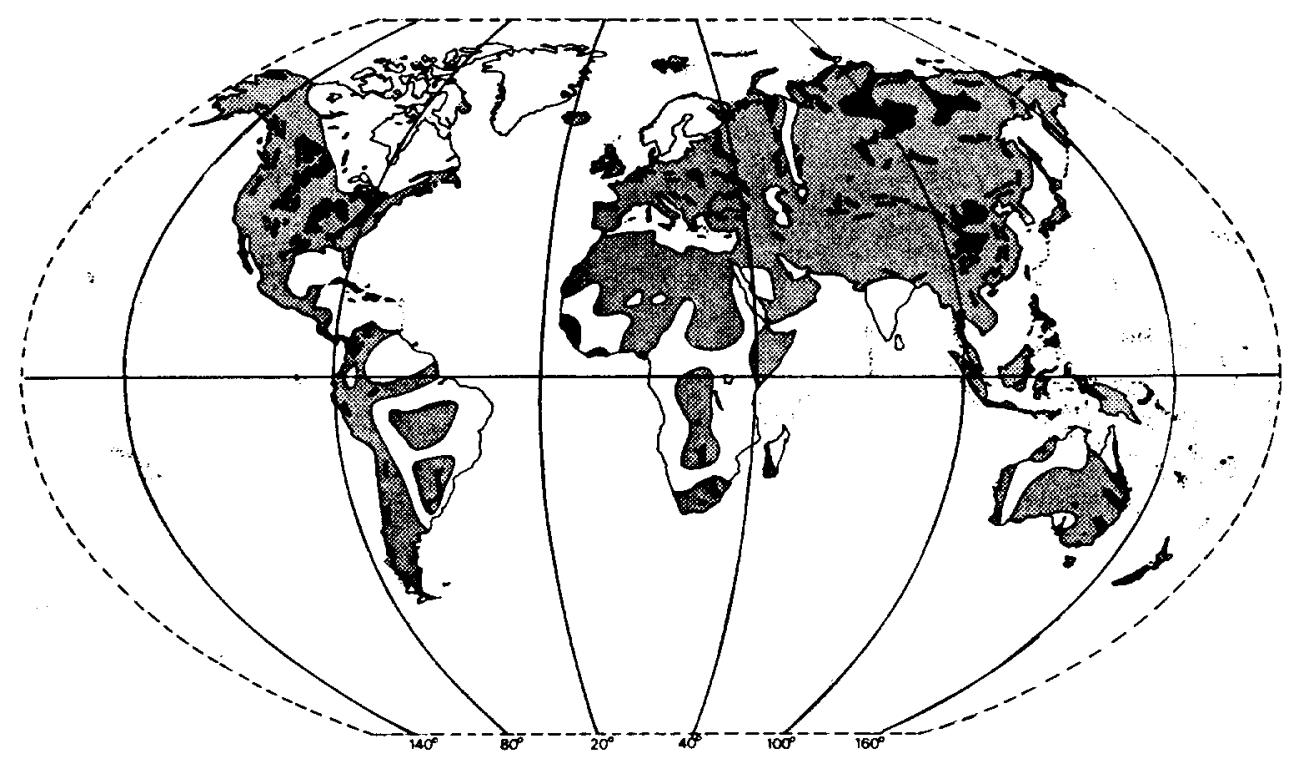

\section{FIGURE 1. Geographic Distribution of Coal Reserves throughout the World ${ }^{1}$}

containing the sulfides of molybdenum, cobalt, nickel, iron, or tin have been particularly studied $[2,4,10]$. Although important principles have been established, there is general agreement that new catalyst formulations and impregnation techniques are required to achieve high activities under low-severity conditions that will make coal liquefaction economically attractive. The goal of this proposal is to develop a technique which will enhance the adsorption, dispersion and liquefaction activities of organic-soluble molybdenum compounds by increasing coal surface hydrophobicity via pretreatment with surfactants. The potential applications of these technologies are considerable; however, it is necessary to explore solutions that will make these processes economically competitive with the price of petroleum. 


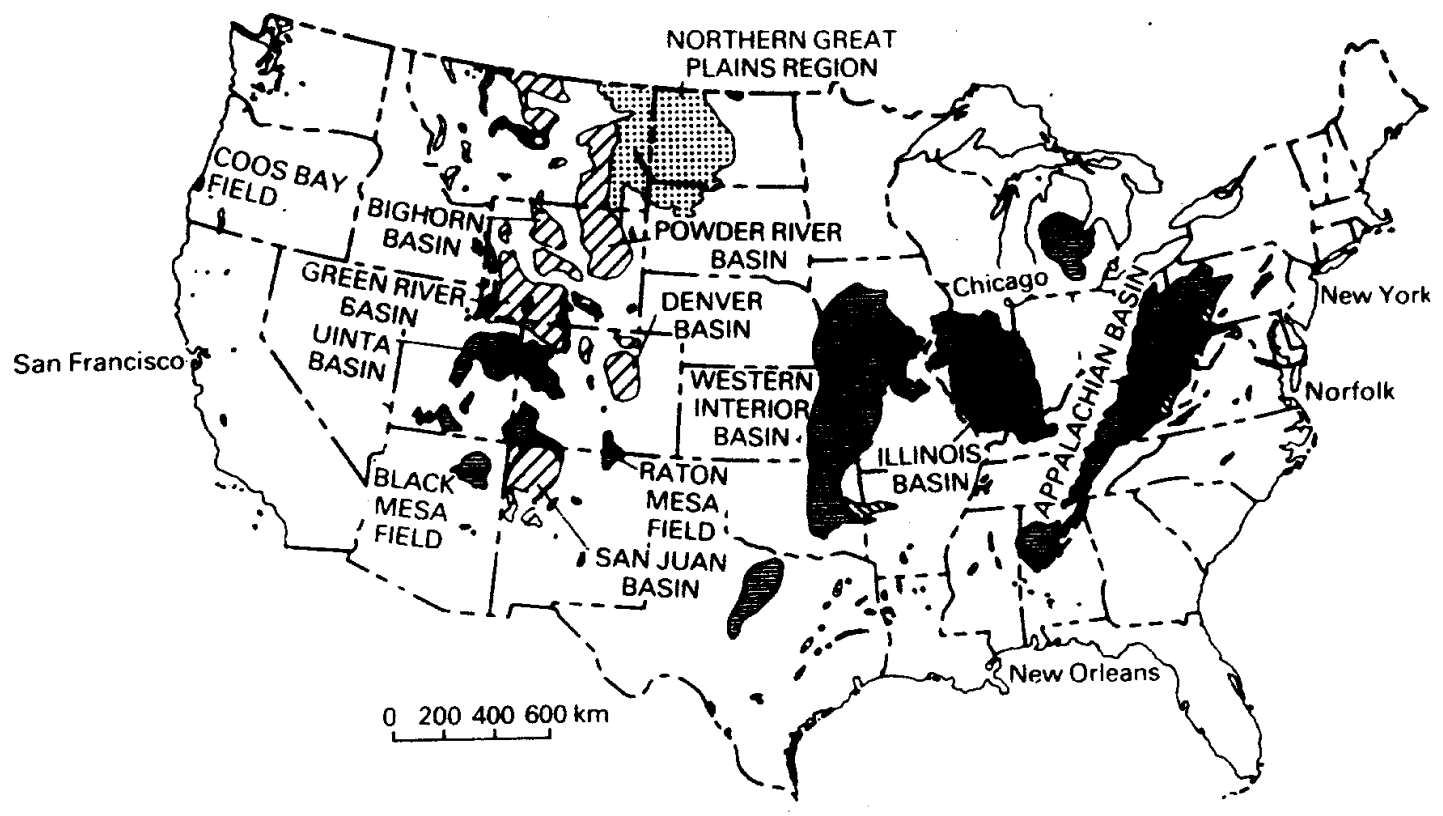

FIGURE 2. Distribution of Coal Fields within the United States ${ }^{1}$

\subsection{PROJECT OBJECTIVES}

\subsection{Research objectives}

Molybdenum catalysts are promising for coal liquefaction on a commercial scale. However, for this potential to be realized, improved techniques must be developed for loading coal with the catalysts since it is well established that the method of catalyst introduction into coal plays a significant role in catalyst performance. An important parameter that has not been previously investigated in catalyzed coal liquefaction is the influence of coal surface hydrophobicity or hydrophilicity on the interaction and uptake of catalyst precursors, even though there is consensus that interfacial phenomena are involved. We propose to conduct systematic and detailed investigations on this fundamental and crucial aspect of dispersing catalysts in coal prior to liquefaction.

The principal objective of the proposed work is to develop a technique, based on coal surface properties, for highly dispersing molybdenum complexes in coal and to 
investigate the application of these compounds as cost-effective catalysts for coal liquefaction.

The specific aims of the project were to:

(1) Increase the surface hydrophobicity of coal by modifying its surface chemical properties through preadsorption of surfactants. This will facilitate maximum catalyst precursor with coal interaction and ensure maximum adsorption and dispersion of the molybdenum catalysts.

(2) Measure the surface charge on selected coals using electrophoresis.

(3) Investigate the surface properties of the coals after pretreatment with various surfactants.

(4) Determine the optimum solution conditions, such as $\mathrm{pH}$ and surfactant concentration, for the most effective catalyst adsorption of the molybdenum catalysts.

(5) Assess the dispersion of the catalysts in the coal.

(6) Determine the liquefaction activities using the proposed catalyst loading technique.

To meet the project objectives the proposed work was subdivided into the following tasks.

(1) Selection and procurement of the coal samples and needed chemicals.

(2) Measurement of the surface charge densities (zeta potentials) of the parent coal as a function of $\mathrm{pH}$.

(3) Analysis of the surface area of the parent coal. 
(4) Treatment of samples of the parent coal with surfactant solution.

(5) Characterization of the treated samples using surface area analysis and zeta potential electrophoretic mobility measurements.

(6) Determination of the interaction of the surfactants with the coal surface using infrared spectroscopy and atomic force microscopy.

(7) Loading of the coal with the catalyst, ammonium molybdate (VI) tetrahydrate (AMT), using ion exchange technique.

(8) Investigation of the effects of molybdenum on the surface of the coal using the surface area analyzer, an atomic absorption spectrometer, and an atomic force microscope.

(9) Measurement of the luminescence intensity to provide supporting evidence for the liquefaction study.

(10) Conduction of liquefaction experiments to determine the efficiency of the proposed technique. Tetrahydrofuran (THF) extraction of the solubilized sample and extraction of the THF soluble products in heptane were performed.

(11) Utilization of UV-Vis measurements to create an adsorption isotherm of the surfactant treated coal (Triton X-100), which should provide information regarding the validity of the liquefaction results.

(12) Analysis and evaluation of the results and preparation of reports.

\subsection{Educational Objectives}

Clark Atlanta University (CAU) is strongly committed to catalysis, energy and fossil fuel research and the proposed project was to have a major impact on the Catalysis and Separation Science (CASS) Center. The catalysis aspect of the CASS center covers catalyst development, characterization and testing.

The proposed project was to enhance the University's master plan for developing undergraduate and graduate degree programs in catalysis science and engineering. This 
would accelerate the University's efforts towards increasing minorities in these fields and contribute to the manpower development in the areas of fossil fuels.

The project was to assist in training students towards the Master of Science or Doctoral degree in Chemistry and for educating undergraduate chemistry and engineering students. Engineering at CAU is a new program and was to benefit greatly from the proposed collaborative project. This type of project provides students at CAU with experience in the chemistry and chemical engineering fields. The project was to expose students to energy research and development and motivate them to pursue careers in catalysis science and engineering. It was also to provide specific science and engineering skills in the design and execution of research, data acquisition and analysis, preparation of technical reports and information transfer, which was to provide the foundation for further education in science and engineering. Eminent scientists and engineers from the petroleum industry were to be invited to participate in the project (e.g. through technical advice and seminar presentations). Such activities were to bring together students and industrial scientists and engineers, enlighten the students as to career opportunities, and provide industry with quality minority students as potential employees. Presentations of the research findings at national chemical and engineering (ACS and AIChE) conferences by the students were to be encouraged as part of their training. These efforts were to contribute to ensuring a future supply of coal scientists and technologies.

\subsection{Impact of the proposed research}

It was expected that the proposed work would make major contributions to the development of a cost-effective technique for highly dispersing molybdenum catalysts in coal's hydrophilic and hydrophobic sites through the adsorption of the coal conversion catalysts. The successful execution of the proposed work was to provide economic benefits by reducing the amount of the catalyst precursors required for coal liquefaction. This was to offset surfactant cost and make the process economically attractive and

competitive. Appropriate selection of the surfactants used for coal-water slurry preparation should allow successful integration of the proposed catalyst loading 
technique into the coal-water slurry mode of transport which will allow sufficient time for catalyst penetration and dispersion in the coal. Catalyst recovery and recycle will prolong catalyst life and further reduce cost. Such improvements would allow the prices of coal-derived liquids to be competitive with those of crude oils and make commercial coal liquefaction economically attractive. The availability of coal-based liquids will offset the shortfalls in future crude oil supplies, as predicted by a recent report [19]. Enhancements in catalyst performance will also be beneficial for the upgrading of heavy oils as well as co-processing of coal and oils.

The project was to provide the opportunity for collaborative work with industry and enhance the education, research and training of minority students in fossil energy and catalysis. The project was also to contribute to ensuring a continuous supply of coal scientists and technologists for the long-term, large-scale development of coal liquefaction as transportation fuels from the nation's large coal reserves.

\subsection{SCIENTIFIC BACKGROUND}

\subsection{Coal Structure and Properties}

Coal is a sedimentary rock formed from the degradation of plants and various mineral matter $^{2}$. Gradual physical changes in the coalification environment such as increases or decreases in temperature and pressure over time determine the characteristics of each coal sample. These characteristics affect the chemical composition, appearance, and economic uses for each type of coal bed. The initial material formed from this process is called peat. A coal sample that has been mildly affected by environmental factors which occur during the decomposition of the plant debris, and has qualities most like peat, is called "soft" or "low rank" coal. On the contrary, samples that have been affected strongly by changes in the environment may be called "hard" or "high rank" coal [1]. Coal samples may be ranked from low to high in the following order: lignite, subbituminous, bituminous, semi-anthracite, and anthracite. 
Today, coal is commonly classified using Seyler's method, which is based upon the carbon and hydrogen contents of the coal sample. Low ranking coals have lower carbon content and higher hydrogen content than higher-ranking coals. On the contrary, anthracites have little hydrogen content and a greater carbon content. Coals of different ranks are capable of producing different amounts of energy. For example, a greater mass of soft coal is required to produce the same amount of energy that a smaller mass of a higher ranking coal may produce [1].

When reporting data from experiments involving coal samples, it is customary to report the proximate and ultimate analysis of the coals used. Proximate analysis provides information regarding the relative amounts of volatile and non-volatile organic components, the percentage of water, and non-combustible mineral materials present. The ultimate analysis provides the elemental composition of carbon, nitrogen, hydrogen, oxygen, and sulfur. For example, Tables 1 and 2 give comparisons of different ranks of coal based upon proximate and ultimate analysis, respectively, from a study performed by Abotsi and co-workers [21].

As evidenced in Tables 1 and 2, the fixed carbon content increases as the coal rank increases. It should also be noted that the oxygen content decreases as the coal rank increases. This factor is important because the electrokinetic properties of coal are dominated by the surface oxygen functionality.

TABLE 1. Proximate Analysis of Some Coals [21] (wt \% as received)

\begin{tabular}{|l|l|l|l|l|}
\hline Coal & Moisture & Volatile Matter & Fixed Carbon & Ash \\
\hline Lignite, PSOC 1482 & 34.5 & 28.2 & 31.8 & 5.6 \\
\hline $\begin{array}{l}\text { Subbituminous, PSOC } \\
1485\end{array}$ & 25.4 & 27.4 & 38.7 & 8.5 \\
\hline Bituminous, PSOC 1493 & 9.4 & 34.3 & 42.5 & 13.7 \\
\hline
\end{tabular}


TABLE 2. Ultimate Analysis of Some Coals [21](wt \%, daf*)

\begin{tabular}{|l|l|l|l|l|l|}
\hline Coal & Carbon & Hydrogen & Nitrogen & Sulfur & $\begin{array}{l}\text { Oxygen } \\
\text { (diff) }\end{array}$ \\
\hline Lignite, PSOC 1482 & 71.3 & 4.5 & 1.1 & 0.8 & 22.2 \\
\hline $\begin{array}{l}\text { Subbituminous, PSOC } \\
1485\end{array}$ & 75.8 & 5.3 & 1.2 & 1.0 & 16.8 \\
\hline Bituminous, PSOC 14934 & 78.1 & 4.9 & 1.5 & 5.9 & 9.6 \\
\hline
\end{tabular}

* daf- dry ash free

Lignite, or brown coal, is a class of coal believed to have been formed from the sedimentation and decomposition of peat. This was followed by the gradual formation of subbituminous and bituminous coals, which should contain 70-80\% carbon. Each type of coal has a unique chemical structure, which is due to the formation or elimination of polar substituents such as hydroxyl and carboxyl groups.

The structure of a coal sample is largely dependent upon the rank of the coal. Low rank coals, such as the bituminous coal used in our experiments, are characterized by their large molecular weights, fewer and smaller aromatic rings, and many carboxylic and phenolic groups. Structures of this type of coal emphasize hydrogen bonding, ion bridges between large molecules and charge transfer interactions.

\subsection{Coal Surface Chemistry}

To determine techniques and methods that may be instrumental in improving many of the coal liquefaction processes, it is extremely important to evaluate each coal sample and analyze its structure and reactivity. Many structural models of the coal molecule exist which aid researchers in developing new technology related to coal liquefaction and gasification. Due to the heterogeneity of coal, no single structure exists [22]. Figure 3 is an example of the molecular structure of a bituminous coal, which provides an illustration of the functionality of a coal sample. 
Many instrumental methods may be used to determine the chemical nature of the surface of a coal sample [23]. However, infrared spectroscopy has proven to be the most useful in providing a wealth of information regarding the functional groups present on the coal surface. Based on these studies, sulfur, nitrogen and oxygen were found to be the three most common elements, with oxygen being the most abundant. This provides reason for the effects of oxygenated groups on physical and chemical interactions on the coal surface. There are a number of aromatic, aliphatic, and heteroatomic components present on the surface, which provide a variety of polar and nonpolar adsorption sites to which a surface-active agent may bind. This heterogeneity contributes to the dual hydrophobichydrophilic character of the coal surface.

Interactions on the coal surface occur frequently via van der Waals forces with most molecules. Few polar molecules have the ability to interact specifically with the surface. For example, the adsorption of surfactant molecules on the coal surface may occur via van der Waals attractions between the nonpolar portion of the surfactant molecule or by hydrophobic bonding. This produces an orientation of the surfactant molecule in which the hydrophilic segment is oriented towards the aqueous solution, which minimizes interfacial tension.

It has been shown that the carboxylic and phenolic species largely determine the surface chemistry of the coal [24-26]. These functional groups, which are formed as a result of oxidation, may increase the hydrophilicity of the coal surface [27]. In aqueous solutions, the dissociation of these strongly acidic carboxylic acid functional groups are easily promoted. The coal surface attains an overall negative surface charge. In a basic medium, coal particles will acquire a net negative charge due to the removal of protons from these species. On the contrary, the dissociation of these species in acidic solutions will only protonate the acidic functional groups, thereby reducing, but not eliminating the overall negative charge density. Thus, coal surfaces usually possess a negative charge in aqueous solution [23]. The following chemical equation illustrates the above point.

$$
\begin{aligned}
& \mathrm{H}^{+} \quad \mathrm{OH}^{-} \\
& \mathrm{R}-\mathrm{COOH}_{2}^{+} \leftarrow \mathrm{R}-\mathrm{COOH} \rightarrow \mathrm{R}-\mathrm{COO}^{-}+\mathrm{H}_{2} \mathrm{O} \\
& \text { ACIDIC BASIC }
\end{aligned}
$$




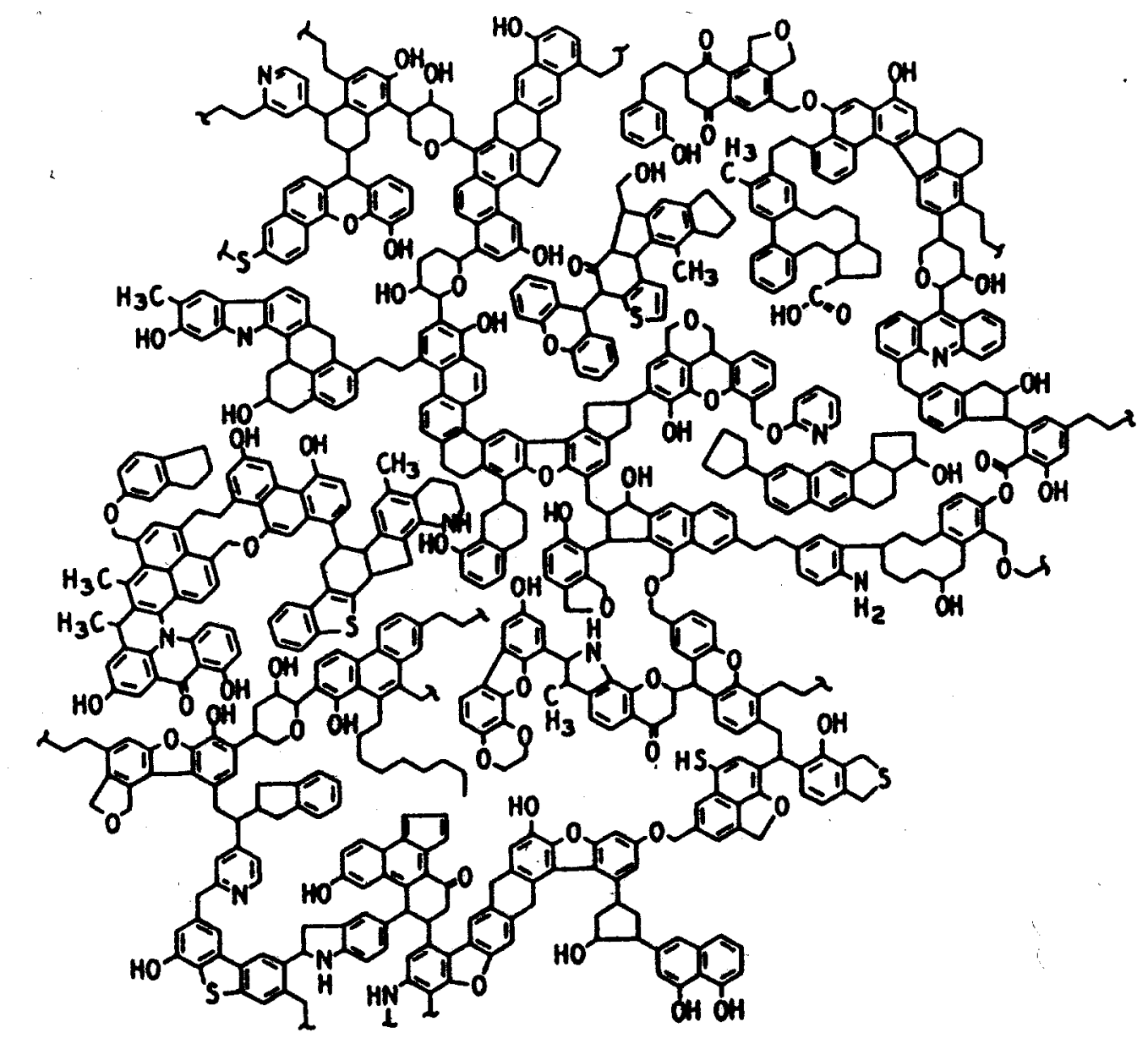

FIGURE 3. Molecular Structure of Bituminous Coal

Due to the fact that there are many scenarios which may determine the overall coal surface charge in solution, it is important that we gain knowledge regarding the coal surface in an effort to effectively adsorb catalysts onto the coal surface for efficient catalyst dispersion. The type and magnitude of the charge on coal can be determined from electrophoretic mobility measurements, or zeta potential measurements. This technique involves suspending coal particles in an aqueous solution contained in a chamber to which an anode and a cathode are connected. Upon application of an electric 
field, a potential is created between the two electrodes and the particles will migrate to the anode or the cathode, depending upon the electrical charge. The electrophoretic mobility is proportional to the charge density on the coal particles and can be displayed by the instrument or calculated by hand.

Assessing the physical structure of the coal surface is as important an analysis as studying the surface functionality. Surface area and pore size distributions provide us with valuable information regarding possible sites for interaction. These physical properties may be studied by the adsorption of gases on the coal surface. The two most commonly used gases are carbon dioxide and nitrogen at very low temperatures. Based on studies by Schmidt [23], three types of pore structures are discernible: macropores, which have dimensions that are greater than or equal to $50 \mathrm{~nm}$; mesopores, pore sizes 2-50 nm; and micropores, which have dimensions less than $2 \mathrm{~nm}$.

Prior to treating the surface of the coal with surfactants/catalysts it is helpful to evaluate the abundance of possible sites for interaction. This can be done by surface area analysis. For example, due to the size of surfactant molecules, they can only penetrate macropores. It should be noted that macropores usually only contribute less than $1 \%$ of the overall surface area of the coal. By performing the surface area analysis, it is possible to estimate the possibility of interaction between surface-active agents and the coal surface.

\subsection{Coal Liquefaction History}

Friedrich Berguis was the inventor of the high-pressure coal hydrogenation process during the early 1900[28]. Coal hydrogenation involves the conversion of coal to petroleum by reacting coal with hydrogen gas at high temperature and pressure. Berguis and his counterparts possessed an awareness of the growing economy and need for gasoline due to the emerging automobile industry. In 1910, he began his study of the cracking of heavy oil residues into gasoline [28]. Berguis' study convinced many of his colleagues that the current processes being used were highly inefficient due to the large loss of hydrocarbons and the coke deposits which often formed on the insides of the walls 
of the reactors. By 1913 he introduced a method to eliminate many of the problems associated with petroleum processing.

In the 1930s Berguis and his colleagues introduced the catalytic hydrocracking process. He was able to demonstrate that small amounts of metal oxides were useful in catalyzing the hydrogenation of coal. He attributed this to the fact that oxides have an ability to hinder the polymerization of hydrocarbon fragments, which may be produced upon heating coal. In addition to this, his research group also conducted successful searches for catalysts, which were useful in each phase of the hydrogenation process. Bergius' main focus was attempting to develop a hydrogenation technique, which did not involve catalysts ${ }^{10}$.

The two-stage coal liquefaction process was developed by Farben of Germany in the late 1920s based upon the research of Berguis[20]. The gasification was completed using brown coal. In order to accomplish this, he divided the well-known gasification process developed by Berguis into two steps. The first phase of the process was the hydrogenation of the solid coal to form a liquid product, followed by the subsequent vapor phase reaction [29] (phase 2). During phase one, the coal was ground and suspended in oil. After the addition of the proper catalyst, the mixture was heated to $300^{0}$ C. The vapor phase reaction, which occurred during phase two utilized hydrogen gas at 230 atm [29]. Although molybdenum catalyst was proven to be useful during the liquefaction of the coal, Farben chose to use iron catalysts because of their low cost. The second phase of the hydrogenation yielded 50-70\% gasoline with an endpoint temperature of $165^{\circ} \mathrm{C}$.

The liquefaction process was frequently used in Germany and England to provide fuels during World War II. By 1943, twelve production plants utilized this process to produce an annual total of four million metric tons of synthetic petroleum of which nearly half were useful for high quality aviation and motor gasoline [28]. 
The use of this process in the United States began shortly after World War II. However, in the early 1950s the price of petroleum in the Middle East decreased, and coal ceased to remain a viable resource for energy. The Arab oil embargo of 1973 caused a surge in the price of oil for the United States. As a result, there was resurgence in coal research to find technically and economically feasible processes for producing liquid fuels, which may supplement transportation fuels.

\subsection{Coal Liquefaction Developments}

After World War II, research on liquefaction technology focused on improving reaction conditions and lowering costs. Today, coal may be liquefied at 150-200 atm and $300^{\circ} \mathrm{C}$ $480^{\circ} \mathrm{C}$ depending upon the process. Improvements were made through the discovery of more efficient catalysts [5-18], donor solvents, and improved reactor configurations. Although there have been many developments, a liquefaction process that can supply fuel at a price that is competitive with the price of oil has yet to be developed.

Some of the challenges facing coal researchers today are:

1. Reduction of the amount of coal liquids wasted due to the difficulties in recovering oils from solid waste being generated.

2. Inadequate coal conversion which results in significant amounts of organic waste.

3. The expensive cost of hydrogen which greatly influences the production cost of coal-derived oils. Improved liquefaction processes should minimize the use of hydrogen, thereby reducing the overall price of production.

4. Poor catalyst dispersion causes limited activity during liquefaction and gasification processes. When investing in a catalyst, researchers would like high catalyst 
dispersion, which should increase the utilization of the coal and enhance the overall efficiency of the process.

Many of the problems associated with coal liquefaction may be solved by using multistage catalytic processes involving: pretreatment and dissolution of the coal, catalytic hydrogenation, and solvent regeneration [22].

\subsection{Catalysts and Coal Liquefaction}

The inorganic material present in coal may contain substances that serve as catalysts during the coal liquefaction process [25-27,30-33]. Pyrite is one of the inorganic materials present in coal, which may serve as a catalyst during the liquefaction process. It has been shown that the addition of pyrite to coal-hydrogen mixtures may increase oil production by more than $50 \%$ after $1 \mathrm{~h}$ of reacting at $723 \mathrm{~K}$, compared to the system without pyrite [34]. The effective catalyst activity of pyrite has been attributed to its ability to hydrogenate the process solvent, thereby improving oil yields [33,35].

Iron sulfate and red mud are other iron containing substances which were originally used for catalysis in coal liquefaction in commercial plants during World War II [2, 5-9]. Hematite, ferrous sulfide, magnetite, and hydrated iron oxide, are examples of other common iron based catalysts.

Metal carbonyls have high activities, which may be linked to their ability to decompose and to produce finely divided, high surface area metallic species during liquefaction. In addition to this, their solubilities in organic solvents may enhance their contact with the hydrophobic sites on coal when loaded with such solvents. Carbonyl compounds of molybdenum, iron, tungsten, cobalt, and nickel have been tested for their high activity as catalysts for the liquefaction of Illinois No. 6 coal, which resulted in coal conversions greater than ninety percent, and oil yields greater than thirty-two percent. The highest oil yield was found to be produced from $\mathrm{Mo}(\mathrm{CO})_{6}$, which produced a yield of $57.7 \%$ [25]. 
Salts of molybdenum compounds have also been used as effective catalysts for coal liquefaction. Examples of such compounds include ammonium molybdate and its sulfided derivatives, molybdenum naphthenate and molybdenum hexacarbonyl. Molybdenum catalysts may easily be compared to iron, as they are more active catalysts for coal liquefaction, but are also more costly. A major challenge facing the usage of molybdenum on a commercial scale is the inability to achieve high levels of catalyst dispersion on coal. Hence, improved techniques for molybdenum dispersion on coal are needed for the enhancement of coal liquefaction activity. In an effort to reduce costs associated with the use of molybdenum, several investigations have been conducted that utilized mixtures of molybdenum and iron [36], although the liquefaction activity of iron is less than molybdenum. The availability and low cost of iron can be a major economic incentive for coal liquefaction on a commercial scale.

\subsection{Catalyst Addition to Coal}

Catalyst addition to the surface of coal may occur in many ways. Common methods of catalyst addition to coal include physically mixing solid catalyst particles with coal; the incipient wetness technique, which involves the addition of a sufficient amount of solution containing the catalyst to fill the pore volume of the coal sample; and exchange of catalyst metal ions with the carboxylic and phenolic protons on the coal surface, also known as ion exchange. It should be noted that different techniques may produce different degrees of catalyst loading under similar reaction conditions. Recently, investigations have been conducted to explore options of improving catalyst dispersion, and reducing the amount of catalyst needed to perform reactions, thereby reducing costs, and reducing the amount of catalyst which must be recovered and regenerated [22].

One of the first attempts to disperse solid catalysts on the coal surface involved oil and water-soluble iron mixtures, which were either impregnated, or ion exchanged on the coal surface through interaction with various oxygen functional groups [22]. High dispersion of molybdenum salts is one of the approaches used in an effort to reduce the amount of excess catalyst usage, because of their excellent liquefaction activity, despite the price. 
In an attempt to enhance catalyst dispersion for liquefaction, supercritical carbon dioxide was used as a medium for the dissolution, the first stage of coal liquefaction, and impregnation of Illinois No. 6 coal with $\mathrm{Mo}(\mathrm{CO})_{6}$ [37]. (Carbon dioxide is a well known solvent for supercritical fluid extraction due to its cost, availability and its relative low toxicity.) A separate batch of coal was loaded by physically mixing powdered molybdenum hexacarbonyl, $\operatorname{Mo}(\mathrm{CO})_{6}$, with coal. Liquefaction studies of the samples under similar conditions showed that the yield, as measured by tetrahydrofuran (THF) solubility, was lower for the samples loaded with catalysts in supercritical carbon dioxide than those to which $\mathrm{Mo}(\mathrm{CO})_{6}$ was added as a powder. Supercritical fluid impregnation caused high penetration of the coal by the catalyst $\mathrm{Mo}(\mathrm{CO})_{6}$, which may have hindered contact of the catalyst with the sulfiding medium $\mathrm{H}_{2} \mathrm{~S}$. However, when $\mathrm{Mo}(\mathrm{CO})_{6}$ was added as a powder the coal was less penetrated, and there was increased exposure of the catalyst to the sulfiding medium $\mathrm{H}_{2} \mathrm{~S}$, resulting in the formation of $\mathrm{MoS}_{2}$, thereby causing an observed higher liquefaction activity.

The above mentioned methods of catalyst impregnation are only two examples of many methods used to load coal samples with catalyst precursors. The method of catalyst addition to the coal surface is as important as the type of catalyst used [36,38-41].

For example, it has been shown that iron sulfate serves as a more effective catalyst when impregnated from solution than when it is physically mixed with coal. Prolonged mixing improved catalytic activity of the solid iron sulfate catalyst, but the enhancement was less than that obtained by iron impregnation [13,36,38].

Recently, researchers have explored the use of surfactants and their ability to enhance coal liquefaction activity. Vittal and co-workers have shown that the use of a metal exchanged anionic surfactant as a precursor to highly dispersed coal liquefaction catalysts is useful in increasing product yield of coal samples [42]. The metal exchanged surfactant was oil-soluble and thermally decomposable, and was included in the reaction by mixing with the reaction solvent. The experimental metals were chosen from a group of well-known catalysts including $\mathrm{Co}, \mathrm{Ni}$, and $\mathrm{Fe}$, all of which are known for high 
activity in coal liquefaction. The anionic catalyst used in the study was Aerosol OT-100, and it was exchanged with cobalt, nickel, or iron via liquid ion exchange. A bituminous coal (Blind Canyon DECS-17) and subbituminous coal (Wyodak, PSOC 1401) were used for the experiment.

According to their results, the Wyodak Coal had a greater response to the effects of the added catalyst. The nickel catalyst was more effective for the Wyodak coal than the cobalt, producing $48.3 \%$ of hexane converted materials versus $36.2 \%$. It was also shown that $\mathrm{Fe}$ was the least effective catalyst at high concentrations $(0.26 \mathrm{wt} \%)$. On the contrary, the nickel and cobalt forms of the surfactant produced catalysts that were active even at low concentrations $(0.065 \mathrm{wt} \%)$. Molybdenum has proven to have a higher catalytic activity than $\mathrm{Fe}, \mathrm{Co}$, and $\mathrm{Ni}$, and under the same reaction conditions, may prove to yield a higher production of hexane converted materials [39].

Coal is a complex heterogeneous solid consisting of organic matter, primarily in the form of materials, derived from plant debris $[43,44]$. It also contains inorganic materials, some of which act as catalysts during coal liquefaction [23,30-32,45-48]. One inorganic constituent of coal, which has received much research attention, is pyrite $\left(\mathrm{FeS}_{2}\right)[2,33,45$ 50]. From investigations on about 75 minerals and additives for coal liquefaction, it has been shown that addition of pyrite to coal hydrogen mixture in a suitable solvent can increase oil yield by more than $50 \%$ after $1 \mathrm{~h}$ reaction at $723 \mathrm{~K}$ compared to the system without added pyrite [50]. It has also been reported that, under the same reaction conditions, the liquefaction activities of the raw coals were much higher than for the corresponding coals which had been "washed" and therefore contained less inorganic matter. The levels of coal conversion to liquids were restored to those of the original coals after addition of sufficient quantities of pyrite to match the iron concentration in the parent coals [51]. Garg and Givens [33] and Brooks et al. [52] have attributed the activity of pyrite to its ability to hydrogenate the process solvent, catalyze coal hydrogenation, and improve oil yields. 
Various other iron-containing substances such as iron sulfate and red mud have been used for coal liquefaction in commercial plants during World War II [2,4-9] and in pilot-plant tests $[12,13]$. Ferrous sulfide $(\mathrm{FeS})$, hematite $\left(\mathrm{Fe}_{2} \mathrm{O}_{3}\right)$, magnetite $\left(\mathrm{Fe}_{2} \mathrm{O}_{4}\right)$, hydrated iron oxide $(\mathrm{FeOOH})$ and iron pentacarbonyl $\left(\mathrm{Fe}(\mathrm{CO})_{5}\right)$, have also been applied [32-33,3738,48-52]. The catalytic activity in iron-sulfur systems have been ascribed to pyrrhotite (Fe1.xS, where $0<x<x<0.125)$ formed from pyrite or by reaction of iron compounds such as iron oxides with added elemental sulfur, carbon disulfide, dimethyldisulfide, dibenzothiophene or with hydrogen sulfide either introduced into the reactor or generated by coal desulfurization during thermal processing [32-33,45-48].

Molybdenum compounds that have been applied to coal liquefaction include ammonium heptamolybdate and its sulfided derivatives, molybdenum naphthenate and molybdenum hexacarbonyl, $\mathrm{Mo}(\mathrm{CO})_{6}$, [46,53-54]. Compared to iron, molybdenum catalysts are more active but they are also more costly. To reduce catalyst cost, several investigations have been conducted on the addition of small amounts of molybdenum to iron [47]. The high activities of the metal carbonyls have been linked to their decomposition to produce finely divided, high surface area metallic species under liquefaction conditions and to their solubilities in organic solvents which enhance their contact with the hydrophobic sites on coal when impregnated from such solvents. $\mathrm{Fe}(\mathrm{CO})_{5}, \mathrm{Mo}(\mathrm{CO})_{6}$ or their mixtures have been reported as potential catalysts for large scale coal liquefaction [53]. However, their high volatilities [46] dictate that novel impregnation techniques are required for attainment of their maximum effectiveness.

Recently, Wender et al. [47] have shown that a new bimetallic catalyst, $\mathrm{Mo} / \mathrm{Fe}_{2} \mathrm{O}_{3} / \mathrm{SO}_{4}{ }^{-2}$, containing $50 \mathrm{ppm}$ Mo and 3500 ppm Fe gave a 78\% liquefaction activity for Illinois No.6 coal with $80 \%$ selectivity to oils at $673 \mathrm{~K}$. The high activities at these low metal loadings are attributed to inhibition of catalyst sintering by the sulfate anion and to easy penetration of the small catalyst particles into the coal and the coprocessing residuum. 


\subsection{Coal and Surfactants}

Catalyst dispersion on coal is dependent upon the nature of the catalysts and the coal surface. Pretreatment of the coal surface with an appropriate surfactant can enhance catalyst dispersion. Potentially, this ongoing research can result in the development of a low cost technique for efficient dispersion of molybdenum catalysts on coal by preadsorption of surfactants onto coal. This should make the surface hydrophobic, enhance the adsorption and dispersion of molybdenum organic compounds and carbonyls, and thus provide information about the roles of coal hydrophilic and hydrophobic sites on the chemistry of adsorption of coal conversion catalysts. Appropriate selection of surfactants used for coal-water slurry preparation should allow successful integration of this catalyst loading technique into the coal-water slurry mode of transport, which will allow sufficient time for catalyst penetration and dispersion on the coal.

The extent of the hydrophobicity of the coal is dependent upon the physical and chemical changes that occurred during its formation [27]. When added from organic solutions, the catalyst will be attracted to the organic sites on the coal, but repelled by the hydrophilic sites. This may produce an uneven distribution of the catalyst on the coal surface, resulting in poor catalyst dispersion, thereby lowering the liquefaction activity.

Surfactants may be used to assist in increasing the amount of catalyst adsorption on the surface of the coal due to their unique molecular structure. Surfactant molecules are comprised of two distinct portions, one of which has an affinity for the solvent to promote the addition of the molecule to the solution. The other portion is repelled by the solvent and has less affinity for the solvent molecules. These two portions of the molecule may also be described as polar and nonpolar or hydrophilic and hydrophobic. By adsorbing the appropriate surfactants onto the coal, the hydrophilic heads of the surfactants will be adsorbed onto oppositely charged sites on the coal, while the organic portions will be oriented towards the organic solution. This should promote hydrophobic interaction between the hydrophobic catalyst solution and the hydrophobic portions of the 
surfactant molecule. On the contrary, the organic portion of the surfactant can also be adsorbed onto the hydrophobic sites on the coal, with the hydrophilic head oriented towards the aqueous solution. This may also enhance the uptake and dispersion of watersoluble catalyst precursors.

There are four types of water- soluble surfactants: anionic, cationic, nonionic, and amphoteric surfactants. Anionic surfactants are water-soluble salts of long-chain carboxylic acids. This class of surfactants may be categorized into three groups: soaps, sulfonates, and sulfates. They ionize in solution to produce long chain molecules that carry negative charges. Sulfates are one of the oldest types of synthetic surfactants. In this experiment a type of sulfate surfactant, in particular, an alkyl sulfate was utilized. Alkyl sulfates may be synthesized from coconut oil and are often used in the preparation of shampoos.

Cationic surfactants may be placed into two categories. The first category consists of long chain primary, secondary, and tertiary amines. These compounds are water soluble and ionize only in acidic solutions to form long chain cations and a simple salt anion. They also ionize in solution producing a molecule with a net positive charge. The second type of cationic surfactants consists of quaternary ammonium compounds. These compounds will ionize to form long chain cations at all $\mathrm{pH}$ levels. It should be noted that cationic surfactants are more strongly adsorbed from solution than any other type of surfactant. This may be due to the fact that most surfaces carry a net negative charge [55].

Nonionic surfactant molecules do not ionize in solution and produce neither a net positive nor a net negative charge. Finally, amphoteric surfactants also ionize in solution, with the long chain ion possessing either a positive or negative charge, which is dependent upon the $\mathrm{pH}$ of the solution.

Due to the complexity of coal surface and the number of variables present in an adsorption experiment, it is extremely important that the solution conditions such as $\mathrm{pH}$, 
temperature and concentration are monitored. The surface charge properties of coal particles may be used as a guide for determining an appropriate surface-active agent for an adsorption experiment [26,56]. Coal zeta potentials have been used in combination with the adsorption of surfactants to increase the dispersion and stability of coal-water slurries [57].

In addition to studying the proper types of surfactants to utilize, it is also important to monitor the concentration of the surfactants based on Fuerstenau's concept of the hemimicelle[58]. Upon the addition of the surfactant solution to the surface of the coal, an initial layer is formed due to hydrophobic interactions. As this concentration is increased, a bilayer is formed which will result in a hydrophilic outermost layer. This process may continue until the surface becomes saturated. At which point, excess surfactant molecules will not be adsorbed by the surface.

\section{TECHNICAL APPROCAH}

This investigation examines the liquefaction activities of coal after surface modification resulting from pretreatment of coal with surfactants, and the subsequent adsorption of a catalyst precursor. An Illinois No. 6 coal (DECS-24) was selected for the study. Zeta potential, BET, FTIR, Atomic force microscopy, UV-Vis and Luminescence Intensity technologies were used to measure the surface properties and the liquefaction activities of the coal. The details of the experimental methods are given below.

\subsection{Materials}

\subsubsection{Description of the Coal}

The Penn State Sample Coal Bank supplied the Illinois No. 6 coal used in this study. Sample configurations are shown in Tables 3 and 4. 
Table 3. Proximate Analysis [59]

\begin{tabular}{|l|l|}
\hline Parameter & (wt. \% as received) \\
\hline Moisture & 13.2 \\
\hline Ash & 11.6 \\
\hline Volatile Matter & 35.4 \\
\hline Fixed Carbon Content & 39.7 \\
\hline
\end{tabular}

Table 4. Ultimate Analysis (daf* basis)[59]

\begin{tabular}{|l|l|}
\hline Element & wt \% \\
\hline Carbon & 76.19 \\
\hline Hydrogen & 5.32 \\
\hline Nitrogen & 1.32 \\
\hline Sulfur & 6.38 \\
\hline Oxygen & \\
\hline
\end{tabular}

\subsubsection{Reagents and Chemicals}

The influence of metal precursor on catalyst uptake, dispersion and activity was investigated using molybdenum acetylacetonate and molybdenum hexacarbonyl. It is anticipated that the differences in the oxygen content of these coals will produce different surface charge densities which will more clearly delineate the influence of coal surface property on the adsorption of the surfactants and catalyst precursors. The investigations will also be conducted on demineralized samples of the coals. This will minimize the inherent catalytic activity associated with the inorganic content of the coal and eliminate the interference of the inherent iron and molybdenum with those which will be added to the coal. The information obtained for the demineralized derivatives will be compared to those obtained for the corresponding parent coals. The various experimental approaches to the work are detailed below. 
The surfactants: dodecyl dimethyl ethyl ammonium bromide (DDAB) $\left(\mathrm{CH}_{3}\left(\mathrm{CH}_{2}\right)_{11} \mathrm{~N}\left(\mathrm{C}_{2} \mathrm{H}_{5}\right)\left(\mathrm{CH}_{3}\right)_{2}{ }^{+} \mathrm{Br}\right.$, a cationic surfactant, sodium dodecyl sulfate (SDS) $\mathrm{CH}_{3}\left(\mathrm{CH}_{2}\right)_{11} \mathrm{OSO}_{3} \mathrm{Na}^{+}$, an anionic surfactant, and Triton X-100 $\mathrm{CH}_{3} \mathrm{CCH}_{2} \mathrm{C}\left(\mathrm{CH}_{3}\right)_{2} \mathrm{C}_{6} \mathrm{H}_{4} \mathrm{O}\left(\mathrm{CH}_{2}\right)_{2} \mathrm{O}\left(\mathrm{CH}_{2}\right)_{2} \mathrm{OH}$, a neutral surfactant, were supplied by Aldrich Chemical Company. Ammonium molybdate (VI) tetrahydrate (AMT), was used as the molybdenum catalyst precursor. It was also supplied by Aldrich Chemical Company.

The experimental scheme is summarized as follows:

(1) The coal samples and needed chemicals were acquired.

(2) The surface charge densities (zeta potentials) were measured for the parent coal as a function of $\mathrm{pH}$.

(3) Surface area analysis was performed on the parent coal.

(4) Samples of the parent coal were treated with surfactant solution.

(5) The treated samples were characterized using surface area analysis and zeta potential electrophoretic mobility techniques.

(6) The interaction of the surfactants with the coal surface was studied using infrared spectroscopy and atomic force microscopy.

(7) The coal was loaded with the catalyst, ammonium molybdate (VI) tetrahydrate (AMT), using ion exchange technique.

(8) The effects of molybdenum on the surface of the coal were investigated using the surface area analyzer, an atomic absorption spectrometer, and an atomic force microscope.

(9) Luminescence intensity measurements were conducted to provide supporting evidence for the liquefaction study.

(10) Liquefaction experiments were conducted to determine the efficiency of the proposed technique. Tetrahydrofuran (THF) extraction of the solubilized sample and extraction of the THF soluble products in heptane were performed. 
(11) UV-Vis measurements were utilized to create an adsorption isotherm of the surfactant treated coal (Triton X-100), which should provide information regarding the validity of the liquefaction results.

(13) Analysis and evaluation of the results and preparation of reports.

\subsection{Preparation of the Coal Water Slurries}

Coal water slurries consisting of only the parent coal were prepared by adding approximately $2.0 \mathrm{~g}$ of coal to $25 \mathrm{ml}$ of deionized water. The loading of the surfactants was accomplished by adding 25-ml solutions of either 0.1 M DDAB, SDS, or Triton X100 to $2 \mathrm{~g}$ of the parent coal. A set of five samples corresponding to $\mathrm{pH} 2,6,8,10,12$ and the control sample were prepared at room temperature for the parent coal slurries. Each coal-surfactant combination was prepared according to the following procedure:

The natural $\mathrm{pH}$ value ( $\mathrm{pH}$ without any adjustments) of each slurry was first recorded. After recording the original $\mathrm{pH}$ values of the coal dispersions, $1 \mathrm{M}$ hydrochloric acid and/or $0.5 \mathrm{M}$ sodium hydroxide, were added dropwise to the coal slurry to obtain the desired $\mathrm{pH}$ values (2-12). All $\mathrm{pH}$ measurements were done using the Hanna Instruments Checker $\mathrm{pH}$ meter, which had been standardized at $\mathrm{pH} 4,7$, and 10. The samples were agitated using the Burrel Wrist Action Shaker for 24 hours. After equilibration, the equilibrium $\mathrm{pH}$ values of the samples were recorded. The samples were filtered by gravity filtration, and air-dried overnight. Dried samples were used for zeta potential measurements, surface area analysis and liquefaction experiments.

\subsection{Zeta Potential Measurements}

Zeta potential measurements were conducted to determine the net surface charge present on the coal particles. The zeta potential technique involves suspending coal particles in an aqueous solution contained in a chamber to which an anode and a cathode are connected. Upon application of an electric field, a potential is created between the two electrodes and the particles will migrate to the anode or the cathode, depending upon the 
electrical charge. The electrophoretic mobility is proportional to the charge density on the coal particles and can be displayed by the instrument or calculated by hand.

Coal water slurries for zeta potential measurements were prepared by placing approximately $2.5 \mathrm{~g}$ of the coal sample in $500 \mathrm{ml}$ of deionized water containing $0.04 \mathrm{~g}$ of $\mathrm{NaNO}_{3}$, for ionic strength adjustments. To better disperse the particles, the solution was sonicated for twenty minutes using a Branson 2200 ultrasonic bath. The coal solution was decanted into five flasks each containing $50 \mathrm{ml}$ of solution. After recording the original $\mathrm{pH}$ values of the coal dispersions, $1 \mathrm{M}$ hydrochloric acid or $0.5 \mathrm{M}$ sodium hydroxide solution was added dropwise to all beakers except the control sample, to adjust the pHs to the desired values ( $\mathrm{pH} 2$ to 12). The samples were shaken on a mechanical agitator for 4 hours using a Burrel Wrist Action Shaker. After equilibration, the equilibrium $\mathrm{pH}$ of each sample was recorded. The zeta potential values were measured at room temperature using a Pen Kem Model 501 zeta meter.

\subsection{Surface Area Analysis}

Surface area analysis is one of many methods used for physical characterization of a solid sample. This analysis was used to determine the relative amount of coal surface available for possible surface interactions. When a porous sorbent is exposed to gas, initial adsorption occurs as a monolayer of adsorbate. As the relative pressure is increased, at constant temperature and sample volume, the adsorption of the monolayer nears completion. This is followed by the subsequent layering of condensed liquid in the pores due to an increase in the vapor pressure until a saturation point is reached, at which point the system has reached equilibrium. This state of equilibrium can be maintained until a decrease in the pressure of the system begins, which facilitates the desorption process. This process may be described mathematically according to the BET equation [60], which may be written as: 


$\frac{\mathrm{P}}{\mathrm{V}\left(\mathrm{P}_{\mathrm{o}}-\mathrm{P}\right)}=\frac{1}{\mathrm{~V}_{\mathrm{m}} \mathrm{c}}+\frac{(\mathrm{c}-1)}{\mathrm{V}_{\mathrm{m}} \mathrm{c}}(\mathrm{P} / \mathrm{Po})$

Where $\mathrm{P}=$ pressure, $\mathrm{P}_{\mathrm{o}}=$ saturation pressure at the measured temperature, $\mathrm{V}=$ volume adsorbed, $\mathrm{V}_{\mathrm{m}}=$ monolayer volume and $\mathrm{c}=\mathrm{a}$ constant for a given material. From the adsorption measurements of $\mathrm{V}$ at various $\mathrm{P}$, for a particular gas (e.g., $\mathrm{N}_{2}$ ), a plot of $\left(\mathrm{P} / \mathrm{V}\left(\mathrm{P}_{\mathrm{o}}-\mathrm{P}\right)\right.$ vs. $\left.\left(\mathrm{P} / \mathrm{P}_{\mathrm{o}}\right)\right)$, according to the BET equation, yields a linear relationship with slope (S) and intercept (I) given by:

$$
\mathrm{S}=\frac{\mathrm{c}-1}{\mathrm{~V}_{\mathrm{m}} \mathrm{c}}
$$

and

$$
\mathrm{I}=\frac{1}{\mathrm{~V}_{\mathrm{m}} \mathrm{c}}
$$

From the slope and intercept, the monolayer volume $\mathrm{V}_{\mathrm{m}}$, may be determined as :

$$
\mathrm{V}_{\mathrm{m}}=\frac{1}{(\mathrm{~S}+\mathrm{I})}
$$

For a material with a known or estimated value of $\mathrm{c}$ (usually $<100$ ), the value of $\mathrm{V}_{\mathrm{m}}$ may be determined by the one point method using the BET equation. If the value of $\mathrm{c}$ is not available, since very often $\mathrm{I} \ll<S$ and $\mathrm{c} \cong(\mathrm{c}-1)$, then the monolayer volume, $\mathrm{V}_{\mathrm{m}}$, may be determined from a one point method as :

$$
\mathrm{V}_{\mathrm{m}}=\mathrm{V}\left(1-\mathrm{P} / \mathrm{P}_{\mathrm{o}}\right)
$$


After determining the volume adsorbed, $\mathrm{V}_{\mathrm{m}}$, it may be used to calculate the total surface

area $\left(S_{g}, \mathrm{~m}^{2} \mathrm{~g}^{-1}\right)$. For nitrogen gas with a cross-sectional area of $0.162 \mathrm{~nm}^{2}, \mathrm{~S}_{\mathrm{g}}$ may be determined as:

$\mathrm{S}_{\mathrm{g}}=\underline{\mathrm{V}}_{\underline{\mathrm{m}}} \frac{(\mathrm{STP}) 6.02 \times 10^{23}}{22,400} \times \frac{16.2 \times 10^{-20}}{\mathrm{~W}_{\mathrm{g}}} \mathrm{m}^{2} \mathrm{~g}^{-1}$

where $\mathrm{W}_{\mathrm{g}}$ is the weight of the sample (coal) in $\mathrm{g}$.

This experiment was performed in two trials. During trial one, surface area analysis was performed using the multipoint BET method. All samples (surfactant treated and catalyst loaded) were degassed for four hours using the Micromeritics Flow Prep 600. Nitrogen gas was used to remove impurities during the degassing process. The experiment was performed using the Micromeritics Gemini 2360 instrument at ${ }^{-} 195{ }^{\circ} \mathrm{C}$, the temperature of liquid nitrogen, with helium gas as the adsorbate. The instrument was standardized using kaolinite.

During trial two, approximately $0.2 \mathrm{~g}$ of the parent coal was placed in the sample chamber of a Micromeritics ASAP 2000 and degassed to remove any impurities. This took place overnight to provide the best possible results. A known volume of the adsorbate was then expelled into the sample chamber, and the pressure of the gas was regulated as necessary. A series of measurements were made involving pressure-volume variations until an equilibrium relative pressure was reached. This experiment was repeated using approximately 0.1 grams of coal that was previously washed with deionized water.

\subsection{FTIR of Coal Samples}

Several IR techniques were used to determine the functionality of the coal surface. Transmission experiments provided general information about the functional groups present. To study the effects of the adsorbance of the surfactant, the DRIFTS (Diffuse 
Reflectance Infrared Fourier Transform Spectroscopy) technique was used. This technique is sensitive to surface adsorbed species, and may also be used in conjunction with Raman Spectroscopy to provide useful information regarding surface functionality.

This experiment was divided into two parts. During the first trial, the coal samples were dispersed in infrared grade $\mathrm{KBr}$ that had been dried overnight at $300{ }^{\circ} \mathrm{C}$, and sieved through a 90 micron sieve. The FTIR spectra were recorded on $10 \%$ coal in $\mathrm{KBr}$ using a Nicolet Magna IR Spectrometer 750 at 500 scans.

During the second trial, transmission measurements were made using a Nicolet Magna 860 FT-IR spectrometer. The pellets used for this analysis were prepared using $0.001 \mathrm{~g}$ of coal sample and $0.300 \mathrm{~g}$ of $\mathrm{KBr}$. The mixture was ground and the pellets were pressed using 10 tons of pressure for approximately ten minutes. One hundred twenty-eight scans for each spectrum were collected with a final resolution of $2 \mathrm{~cm}^{-1}$.

\subsection{Atomic Force Microscopy}

Following the surfactant and catalyst loadings of the coal samples, the surfaces of the samples were examined using Atomic Force Microscopy (AFM) at the Savannah River Ecology Laboratory (SREL), Aiken, SC. The instrument used was a Burleigh ARIS-3300 AFM with two sample scanning modes. AFM is a variation of the Scanning Tunneling Microscope, and measures the interatomic forces and surface features. The samples were mounted on an epoxy and imaged by tapping the probe tip of the AFM instrument. Tapping mode imaging overcomes the limitation of conventional scanning modes (contact and non-contact mode), by alternately placing the tip of the scanning probe in contact with the surface to provide high resolution and then lifting the probe tip off the surface to avoid dragging the tip across the surface. The technique allows high resolution topographic imaging of sample surfaces that are easily damaged, loosely held to their substrate, or otherwise difficult to image by other AFM techniques. The surfaces of the samples were imaged in the dimension range from $5 \mathrm{~nm}$ to $100 \mathrm{~nm}$. The imaging of the samples posed some difficulties in the area of uneven topology. Imaging even small 
areas of the particle surface was difficult since the topology was typically greater than the length of the AFM probe tip.

\subsection{Luminescence Intensity Measurements}

Seven coal samples and two solid surfactant samples (SDS, DDAB) were prepared and studied. A random subsample was removed from each container and spread evenly onto the level surface of modeling clay affixed to a glass microscope slide. To secure their position, particles were pressed flat into the clay between glass microscope slides in a mounting press. Relatively flat regions of particles were brought into focus in reflected white light using a Leitz MPV-2 research microscope with an air objective at 625 total magnification. Measurement of luminescence intensity was performed by directing 390$490 \mathrm{~nm}$ light from a mercury-arc lamp onto exposed coal surfaces. An interference filter was used to eliminate light below $510 \mathrm{~nm}$. A light beam at wavelength $600(+/-60 \mathrm{~nm})$ was allowed to impinge on the photomultiplier. The photoelectric system was standardized with respect to the intensity of a uranyl glass. The procedure was repeated for 20 individual particles and the mean luminescence intensity value and standard deviation were determined.

\subsection{UV-Vis Measurements}

UV-Vis Spectroscopy was used to create an adsorption isotherm based upon the concentration of the surfactant solution before and after its adsorption onto the coal surface.

The absorbance of the surfactant solutions were measured by first utilizing Beer's Law, which is stated below:

$$
\mathrm{A}=\mathrm{abc}
$$

where, $\mathrm{a}$ is a proportionality constant called the absorptivity, b represents the cell length, and $\mathrm{c}$ is the concentration. This equation may also be written as follows: 


$$
\mathrm{A}=\varepsilon b c
$$

where $\varepsilon$ represents the molar absorptivity.

Beer's law is useful when describing the absorption of dilute solutions. Equation 10 was to determine the equilibrium concentration of the supernatant. Then this concentration was used to determine the amount of surfactant absorbed according to equation 11 below.

$\mathrm{A}_{\mathrm{a}}=(($ Initial Concentration - Equilibrium Concentration $)$ Volume $) /$ mass of coal

UV spectroscopy measurements were completed using the Shimadzu UV 2100 UV-Vis Recording Spectrophotometer. The measurements were taken in absorbance mode using a medium scan speed, and $1.0 \mathrm{~nm}$ slit width.

Ten surfactant solutions were prepared with concentrations ranging from $1.0 \times 10^{-4} \mathrm{M}$ to $5.0 \times 10^{-3} \mathrm{M}$ Triton X-100. Eleven coal samples weighing approximately $0.2 \mathrm{~g}$ were measured, and $50 \mathrm{ml}$ of surfactant solution was added to each of the first ten samples. As a control, deionized water was added to test its effect on washing coal. The samples were agitated overnight using a Scientific Equipment Products Tube Rotator. The coal samples were then centrifuged at 10,000 rpm for 30 minutes using the Sorvall RC 5B Plus. The supernatant solution was filtered via gravity filtration, and analyzed for its surfactant concentration using the UV-Vis Spectrophotometer.

\subsection{Liquefaction Studies}

The effectiveness of the catalyst loading technique was measured by liquefaction activities of the raw coal and the catalyst loaded samples. Liquefaction studies were conducted in a stainless steel batch microautoclave reactor. The internal volume of the reactor including all tubing and connections was $60 \mathrm{ml}$. The samples were tested using $6.6 \mathrm{~g}$ of solvent, $3.3 \mathrm{~g}$ of coal and 6.9 MPa (1000 psi) ambient hydrogen pressure. For

continuous monitoring of pressure and temperature during the experiment, an internal thermocouple and pressure transducer were used. The reactor was then attached to the 
rocker arm of a motor that vibrated at 180 cycles/min, and then plunged into a pre-heated sandbath that was heated to $425{ }^{\circ} \mathrm{C}$ in 40 minutes. This temperature was maintained for 30 minutes, after which the reactor was removed and cooled with water. The liquid fraction was subjected to extraction using tetrahydrofuran (THF). Heptane soluble products were extracted from the THF extracts. Coal conversions were calculated based on THF and heptane solubility.

\section{RESULTS AND DISCUSSION}

\subsection{Zeta Potential Measurements}

The zeta potential results in Figure 4 show that the parent coal is negatively charged within the $\mathrm{pH}$ range investigated, and that the negative charge density increased with an increase in the slurry $\mathrm{pH}$. This behavior is attributed to the dissociation of the carboxylic and phenolic acid groups which dominate the coal surface [22-24]. In aqueous solutions, phenol and the carboxylic acid functional groups act as weak acids by donating protons. Upon dissociation, these molecules become negatively charged ions. This dissociation is enhanced in basic solution, whereas the surface groups are protonated in strongly acidic solutions. In strongly acidic media, the overall negative surface charge density is reduced as shown in Figure 4.

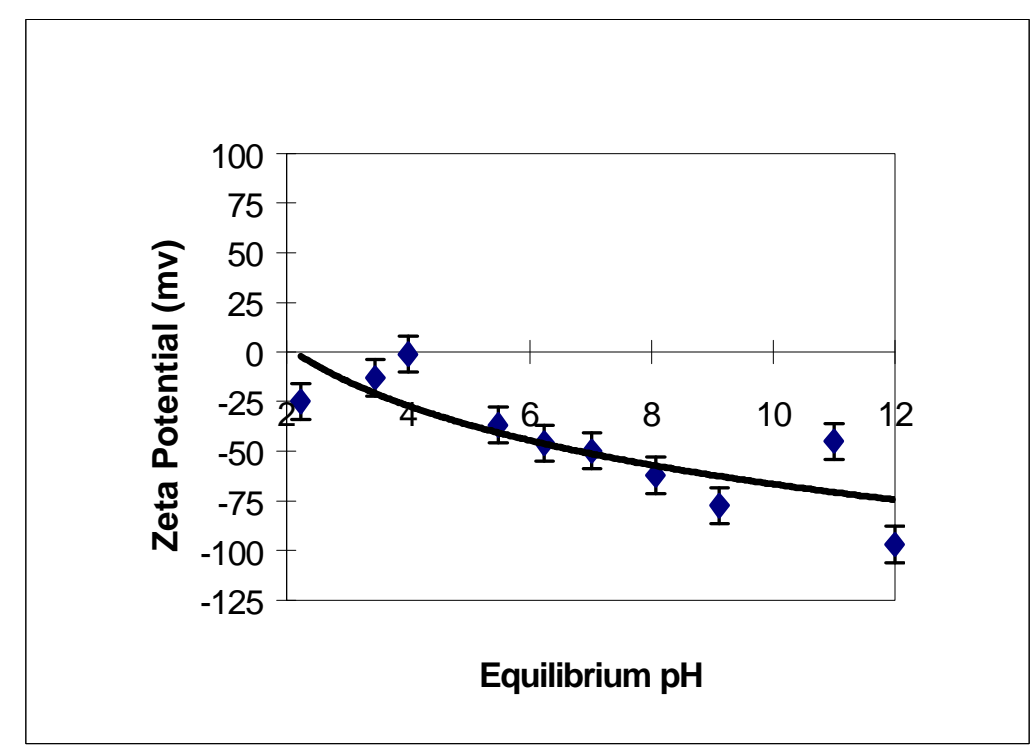

FIGURE 4. Zeta Potential Measurements of the Parent Coal as a Function of pH 
Figure 5 shows the variation in zeta potential of coal samples loaded with increasing concentrations of the cationic surfactant, DDAB. This experiment was conducted to observe the effects of increasing the surfactant concentration, and to evaluate the most effective concentration for surfactant loading. The figure shows that the coal particles generally produced positive zeta potentials in the presence of DDAB. Since DDAB is cationic, there exists a coulombic attraction of the surfactant molecule's polar head, and its subsequent adsorption to the negatively charged sites on the coal surface. When this occurs, the hydrocarbon tail of the surfactant molecule will be oriented towards the solution. This will lead to a reduction in the negative surface charge, at low concentrations.

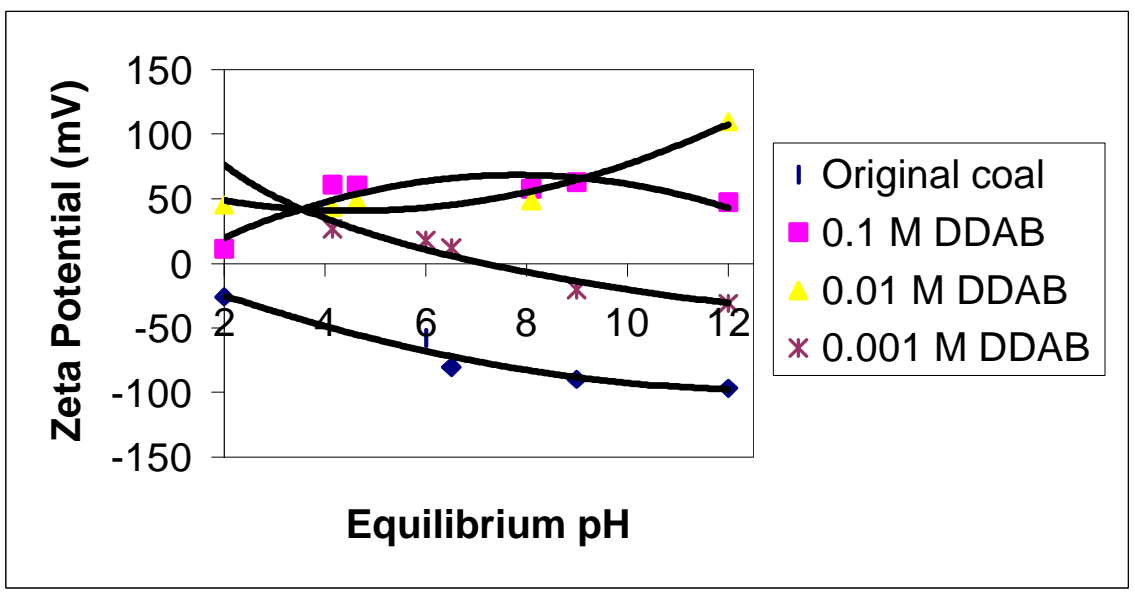

\section{FIGURE 5. Zeta Potential Measurements of Coal Treated with DDAB Surfactant as a Function of pH}

From Figure 5, it was observed that at $0.001 \mathrm{M} \mathrm{DDAB}$, the zeta potentials are positive and become more negative below $\sim \mathrm{pH}$ 8. At 0.01 M DDAB, the zeta potentials are positive within the entire range studied. The zeta potential values increased significantly with an increase in $\mathrm{pH}$ between $\mathrm{pH} 7.5$ and 12. When the DDAB concentration was

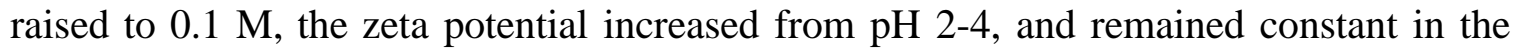
range 4 to 11 . This trend appeared to be more positive in the $\mathrm{pH}$ range 4 to 11 than the other surfactant treated samples. This phenomenom may be attributed to strong DDAB adsorption and possible micelle formation. When the DDAB concentration on the surface exceeded the critical micelle concentration $\left(1.2 \times 10^{-4} \mathrm{M}\right)[25]$ around $\mathrm{pH} 6-8$, the 
molecules formed aggregates with the nonpolar tail of the surfactant oriented towards the interior of the micelle. This may be one possible explanation for the decline in the zeta potential values. As the micelles were formed excess surfactant molecules were not adsorbed by the surface and were therefore expelled into the solution.

Another possible explanation for the trends seen in Figure 4 may be explained by Fuerstenau's hemimicelle concept [14], the increase in concentration of the surfactant contributes to the positive surface charge density due to formation of layers of hemimicelles. In addition to the formation of hemimicelles, nonpolar interactions between the surfactant tail and similar sites on the coal surface may cause interactions which would position the cationic portion of the surfactant molecule towards the solution, leading to an increase in the number of positively charged sites on the coal surface.

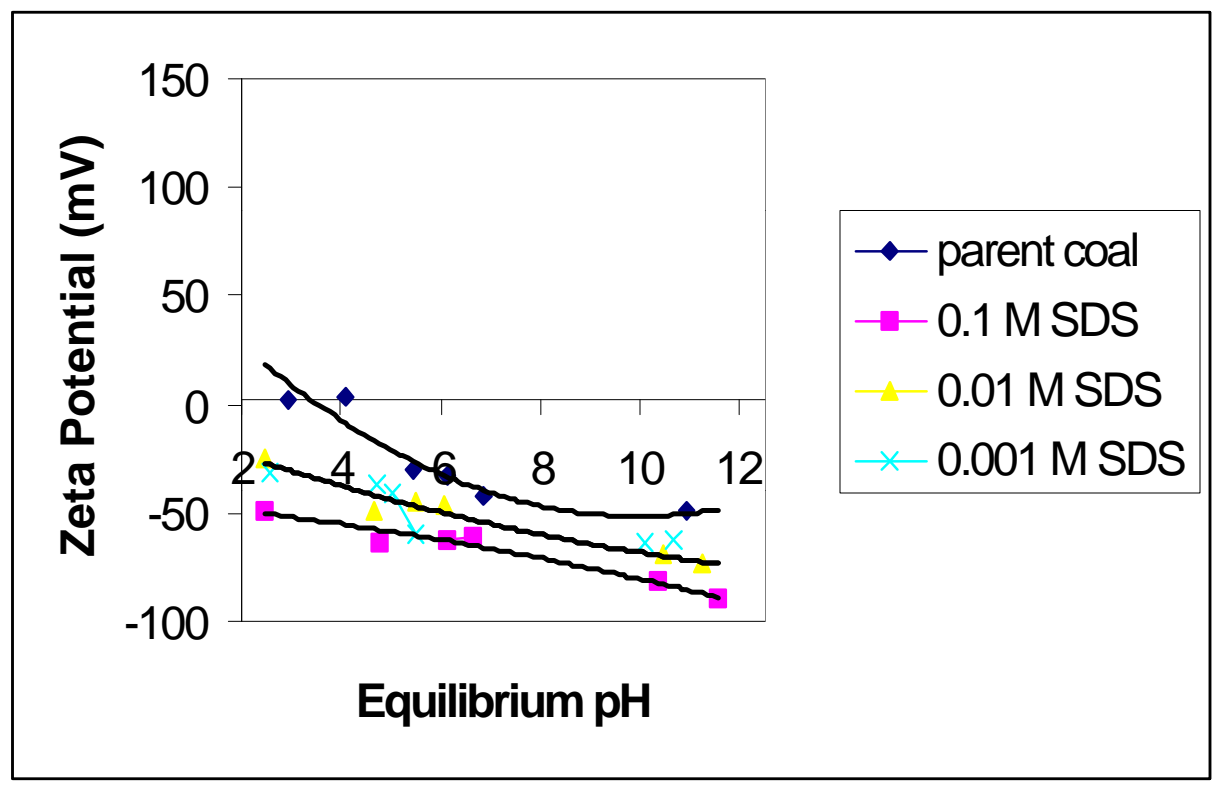

FIGURE 6. Zeta Potential Measurements of Parent Coal Treated with SDS as a Function of pH.

In contrast to the effect of DDAB, the zeta potentials of the coal particles became more negative than those of the parent coal in the presence of SDS, as shown in Figure 6. The negative charge density increased with an increase in the slurry $\mathrm{pH}$ and in the concentration of SDS. Since the surface of the raw coal is negative, the adsorption of 
SDS must occur through the hydrocarbon chain of the molecule, with the anionic head oriented towards the solution.

As shown in Figure 7, the maximum zeta potential of the raw coal was about $-80 \mathrm{mV}$. However, adsorption of 0.25 and $0.1 \mathrm{M}$ Triton reduced the negative charge density appreciably. This may seem surprising since Triton is a neutral surfactant. However, nonpolar interactions between the coal surface and the surfactant molecule account for the change in zeta potential measurements. Surfactant concentration also had an effect upon the reduction of the surface charge density. The stronger surfactant concentration had a slightly greater effect upon reducing the surface charge.

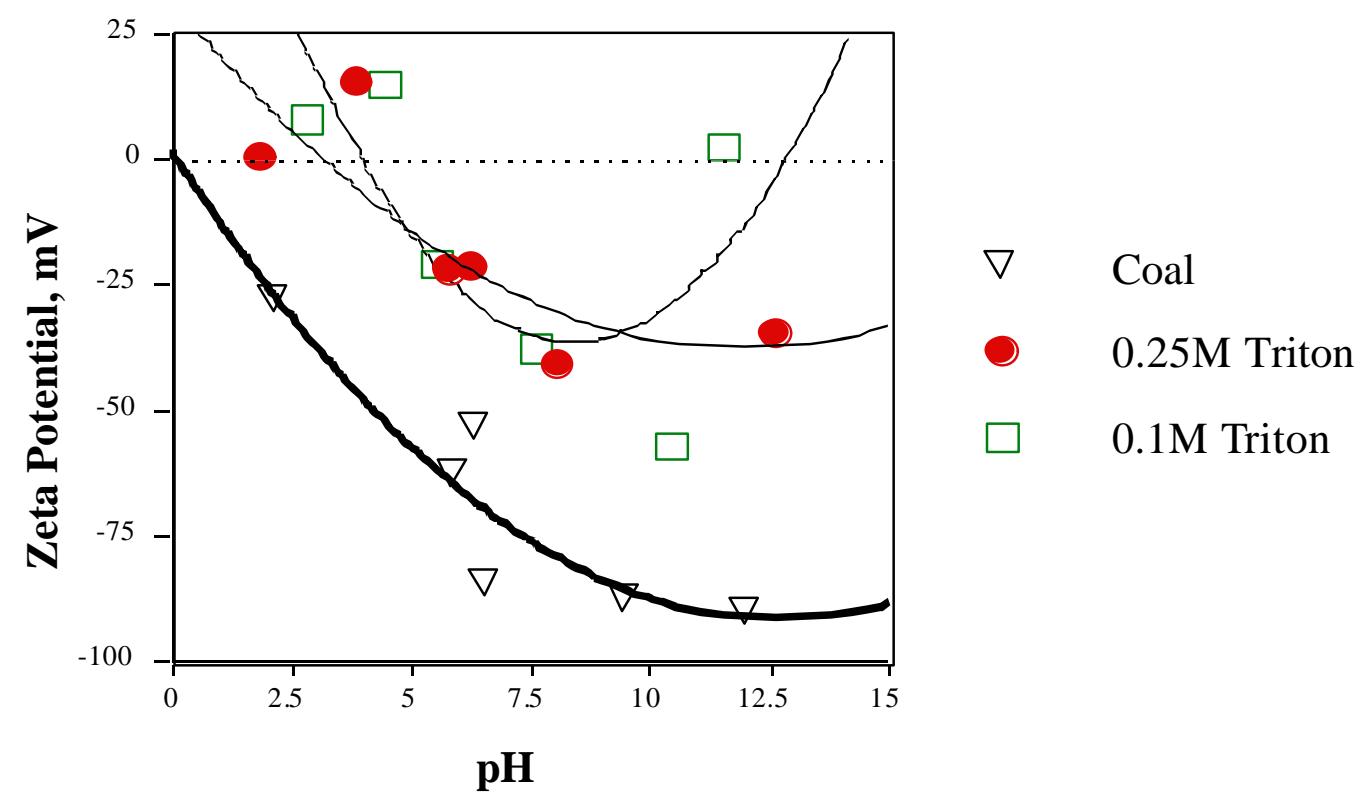

FIGURE 7. Zeta Potential Measurements of Coal Treated with Triton X-100 as a Function of pH

\subsection{Surface Area Analysis}

The results of the surface area measurements are shown in Table 5. The trends displayed are consistent with results from zeta potential measurements and molybdenum adsorption 
experiments. The greater the adsorption of a species (surfactant/catalyst), the lower the surface area.

TABLE 5. Surface Area Measurements of Coal Samples

\begin{tabular}{|l|l|}
\hline SAMPLE & SURFACE AREA $\left(\mathbf{m}^{2} / \mathbf{g r a m}\right)$ \\
\hline Coal & $31.00 \pm 0.45$ \\
\hline Coal + water & $77.23 \pm 0.32$ \\
\hline Coal + Molybdenum & $45.25^{* *}$ \\
\hline Coal + SDS & $18.42 \pm 0.19$ \\
\hline Coal + SDS+ Molybdenum & $34.86^{* *}$ \\
\hline Coal + DDAB & $9.253 \pm 0.99$ \\
\hline Coal + DDAB + Molybdenum & $19.35^{* *}$ \\
\hline Coal + Triton & $9.520 \pm 0.14$ \\
\hline Coal + Triton + Molybdenum & $8.84 \pm 0.24$ \\
\hline
\end{tabular}

\section{** single point determinations - the others are multipoint determinations}

The adsorption isotherm at the gas-solid interface was established according to the BET method. Because the coal surface is porous, the shape of the isotherm differs from that of a uniform solid surface. According to Figures 8 and 9 the adsorption isotherm for the coal surface is characteristic of that of a typical porous solid. The logarithmic nature of the curve is due to the gradual change in the relative pressure of the system. The "knee" in the curve reflects the formation of monolayer coverage of the coal surface. This is followed by the subsequent layering of condensed liquid in the pores due to an increase in the vapor pressure until a saturation point is reached, at which point the system has reached equilibrium.

The measured surface area of the coal surface was $30 \mathrm{~m}^{2} / \mathrm{g}$. The surface area of the coal sample, which was washed with deionized water more than doubled to $77 \mathrm{~m}^{2} / \mathrm{g}$. This is an indication that many of the species present within the coal sample are water soluble, 


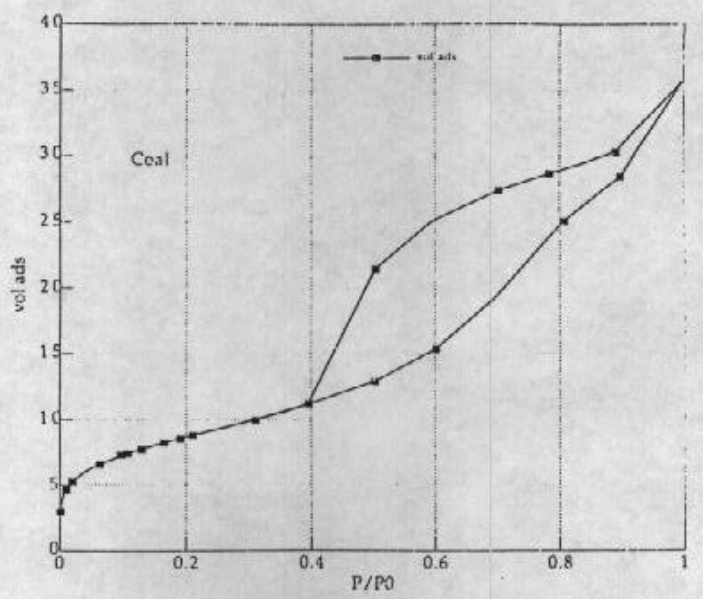

FIGURE 8. Adsorption Isotherm of Parent Coal

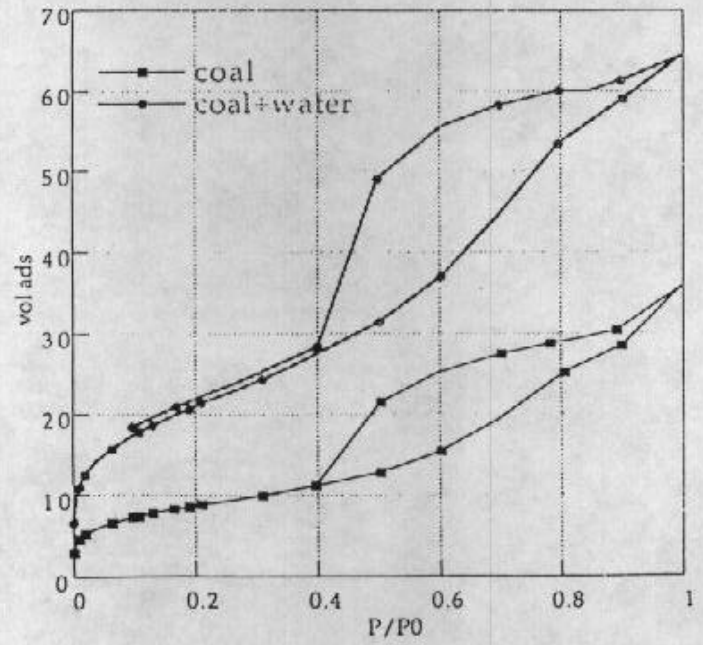

FIGURE 9. Adsorption Isotherm for Coal Treated with Water

and may be loosely bonded. Since surfactant loading takes place in aqueous solution, it is possible that many of the polar surface groups may become easily dissociated from the 
coal surface within solution. This also presents the possibility that coal washing may be one method of increasing the number of active sites available for nonpolar surfactant adsorption. Further investigation of the surface area of surfactant adsorbed coal species shows a decrease in the surface area with an increase in surfactant/catalyst loading. The greater the decrease, the greater the amount of surface coverage due to surfactant loading. This binding may be due to either electrostatic forces or Van der Waals forces depending upon the classification of the surfactant and the amount of surfactant loading.

The adsorption of molybdenum increased the surface area of the parent coal. This increase in surface area may have been due to the method of molybdenum adsorption, which involved exposing the coal sample to an aqueous solution, followed by gravity filtration. This may have had a "washing" effect on the coal surface, causing many of the surface species to have been removed both by physical and chemical interactions.

Coal treated with Triton X-100 had a much lower surface area than the original coal, suggesting a high loading of the Triton surfactant. This may be due to a combination of interactions that may occur between the coal surface and the surfactant molecule as a result of weakly polar and nonpolar interactions.

Finally, the coal surface treated with Triton and molybdenum possessed the lowest overall surface area. However, the adsorption of molybdenum on the Triton treated coal had no significant effect on the surface area. Possible adsorption sites may have been blocked due to surface coverage by the surfactant. This may be an indication of oversaturation by the surfactant leading to inaccessibility of possible adsorption sites.

\subsection{Molybdenum Adsorption of Surfactant Treated Coal vs. pH}

The effects of surfactants on the adsorption of molybdenum are shown in Figure 10. The observed molybdenum adsorption patterns can be explained by evaluating the electronic charges on the coal surface and their interaction with the molybdenum species. In aqueous solution, ammonium molybdate tetrahydrate will dissociate to form various 
molybdenum oxyanions. Thus, the adsorption of the molybdenum species should be promoted by the positively charged sites on the coal created by the adsorption of $0.1 \mathrm{M}$ DDAB.

A strong correlation was observed between the molybdenum adsorption and surfactant treatment of coal particles. As the $\mathrm{pH}$ of the coal slurry containing the surfactant treated coals increased, the amount of molybdenum adsorption decreased for surfactant treated and untreated coals. This may be attributed to a reduction in the amount of protonated species present for molybdenum oxyanion attraction with increasing $\mathrm{pH}$.

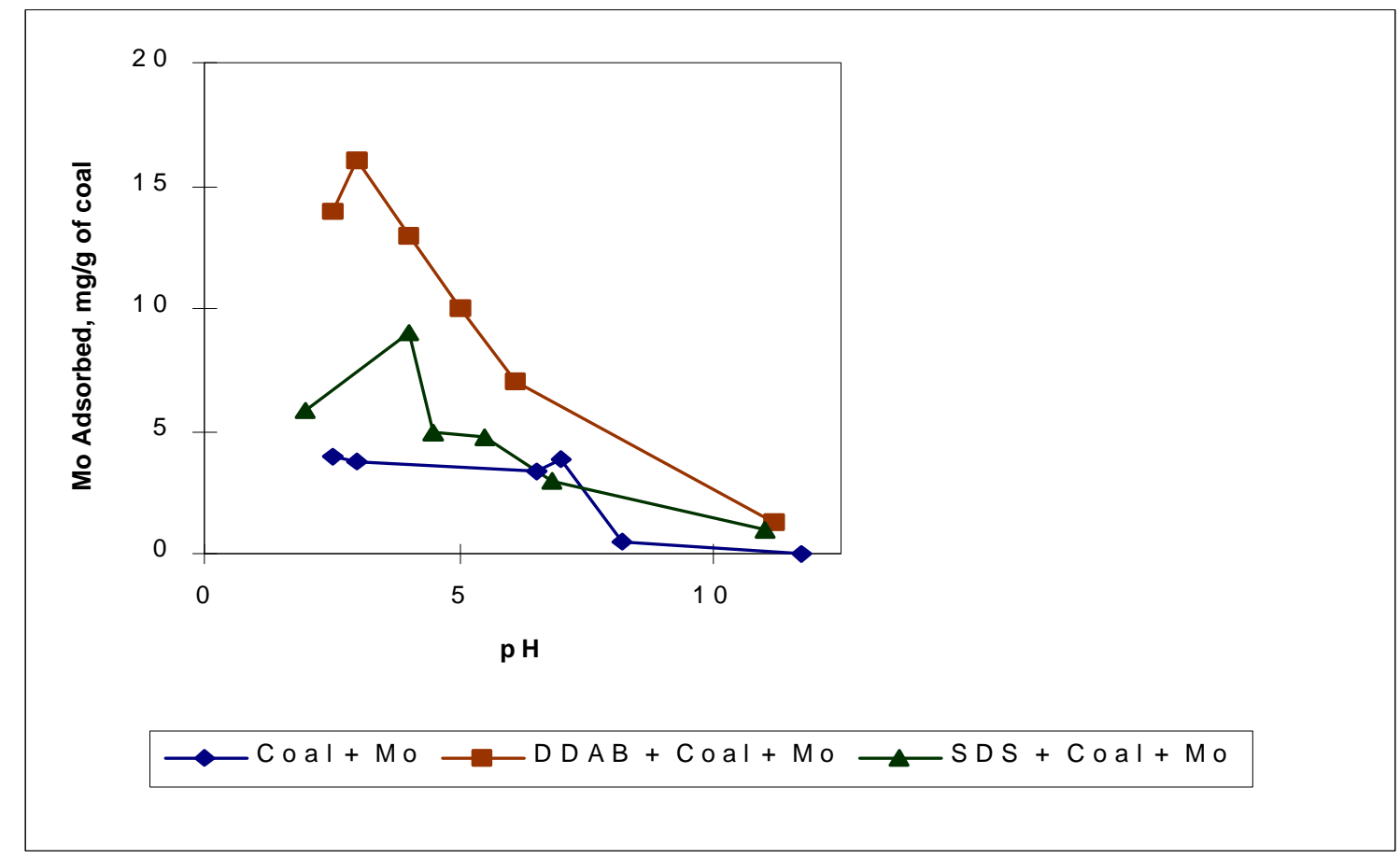

\section{FIGURE 10. Effect of Surfactant and pH on Molybdenum Adsorption of DECS-24 Coal (0.1 M DDAB, 0.1 M SDS, 1000 ppm molybdenum)}

The adsorption of molybdenum oxyanions should occur at those sites which have been protonated within acidic medium. The untreated coal also had an overall negative surface charge, which may have contributed to low molybdenum adsorption due to repulsive 
forces between the catalyst and the surface of the coal. This phenomenon may be observed with coal particles treated with 0.1 M SDS.

At low $\mathrm{pH}$ values, SDS treated particles had a higher molybdenum adsorption than the untreated coal particles. At high $\mathrm{pH}$ values the difference in molybdenum adsorption became less significant.

The coal surface treated with $0.1 \mathrm{M}$ DDAB had a higher molybdenum adsorption than the untreated coal and the coal treated with 0.1 M SDS over the entire pH range. This adsorption $(16 \mathrm{mg} / \mathrm{g})$ occurred at $\mathrm{pH} 3.0$, compared with the adsorption $(9 \mathrm{mg} / \mathrm{g})$ of SDS treated coal at $\mathrm{pH} 4.0$.

This data may be compared to the results of the zeta potential experiments. The zeta potential data of the coal treated with $0.1 \mathrm{M}$ DDAB should correspond to the molydenum adsorption data. With a positive surface charge on the coal, adsorption of molybdenum was expected. The zeta potential values of the $0.1 \mathrm{M}$ DDAB treated coal were positive throughout the entire $\mathrm{pH}$ range. However, there appears to be no significant pattern between the surface charge results provided by the zeta potential measurements, and those of the molybdenum adsorption experiments. Therefore, the adsorption of the catalyst may be promoted by alternative factors.

The effects of surfactant concentration on the adsorption of molybdenum are shown in Figure 11. A higher molybdenum adsorption was attained with the use of DDAB as the surfactant. The minimum amount of this surfactant required to saturate the surface was $0.1 \mathrm{~mol} / \mathrm{L}$. However, the opposite effect was observed when the anionic surfactant (SDS) was used. There was very little adsorption of SDS within the concentration range studied. 


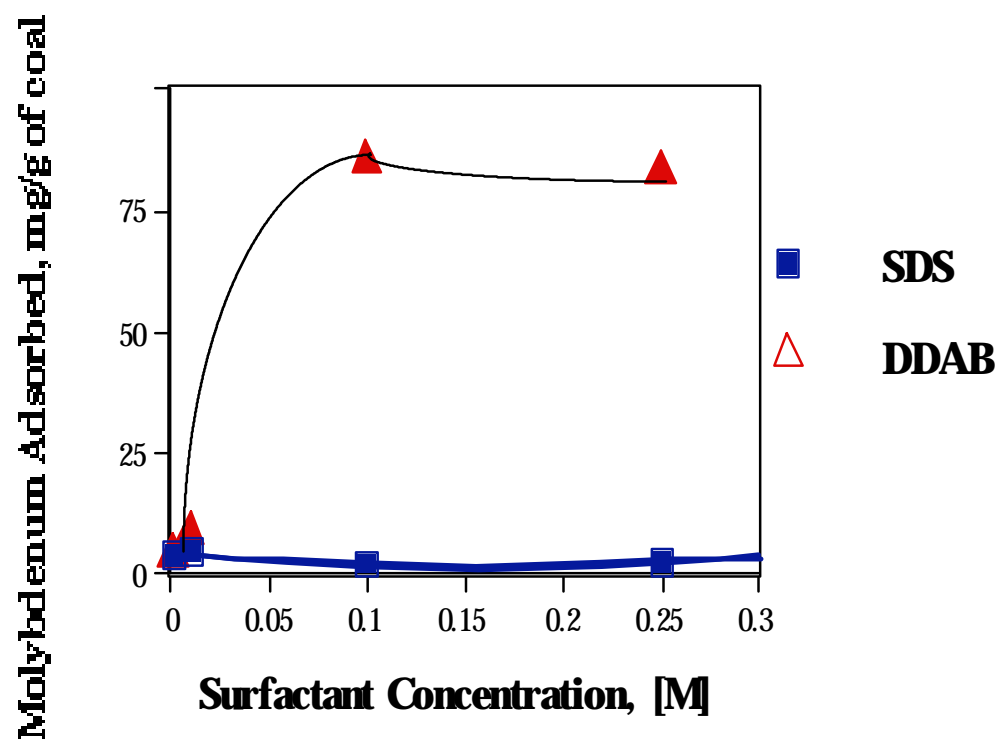

\section{FIGURE 11. Dependence of 1000 ppm molydenum Loading on DDAB and SDS Concentration}

\subsection{FTIR of Coal Samples}

The FTIR spectra of the untreated coal and those onto which molybdenum and DDAB or SDS were adsorbed are shown in Figure 12. The FTIR spectrum for the original coal is provided in spectra $\mathrm{B}$. The $\mathrm{C}-\mathrm{H}$ bands at $2800-3000 \mathrm{~cm}^{-1}$ were used to qualitatively monitor the presence of surfactants. The intensities of these bands are higher for the surfactant treated samples than for the raw coal (B). The intensity of the DDAB treated coal (C) is stronger than those of the SDS treated specimen (D), both of which are stronger than the original coal (B). Therefore, this information provided evidence which confirmed the adsorption of the surfactants onto the coal surface. 


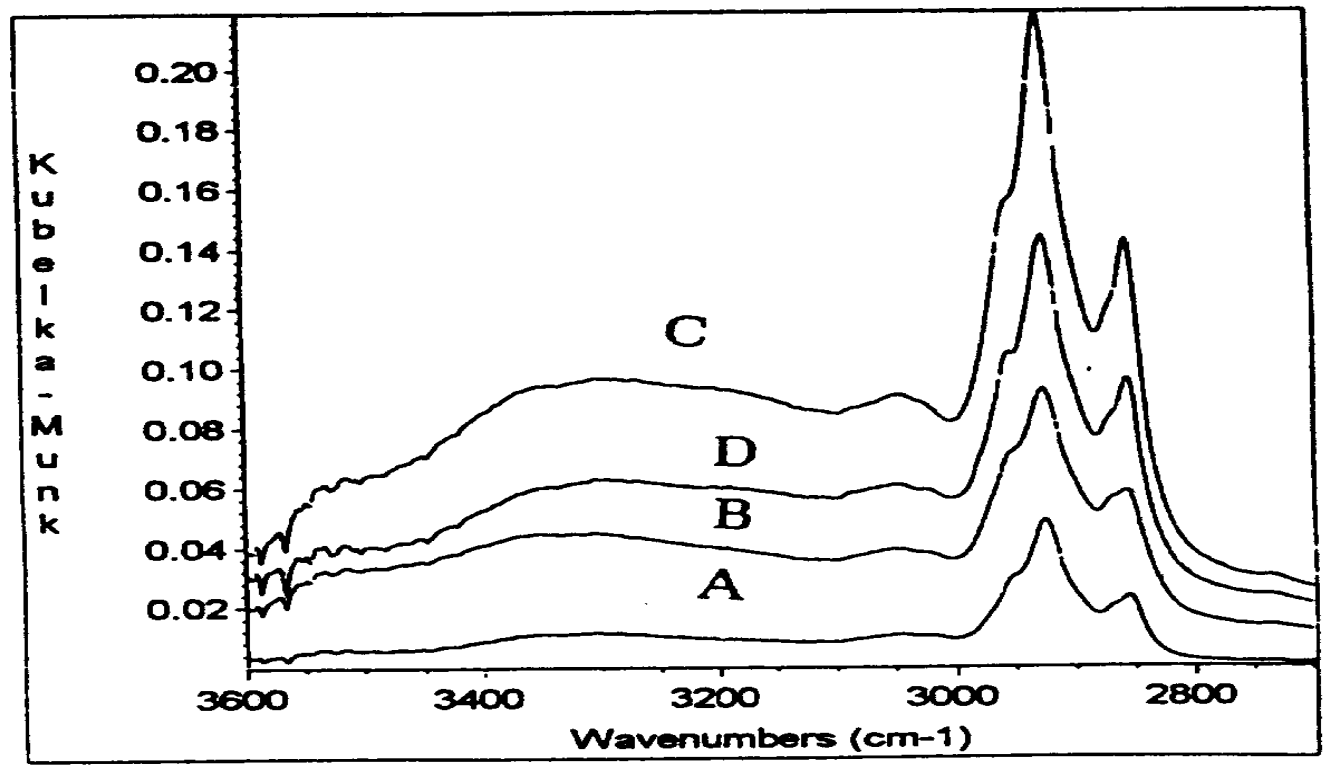

\section{Figure 5. FTIR spectra of the Original and the Surfactant-Treated Coals after Molybdenum Adsorption. A: Coal + Mo; B: Original Coal only; C: Coal + DDAB + Mo; D: Coal + SDS + Mo}

FIGURE 12. FTIR Spectra of the Original Coal and the Surfactant Treated Coals after Molybdenum Adsorption.

\subsection{Atomic Force Microscopy}

The results of the Atomic Force Microscopy studies provided further evidence in support of the adsorption of surfactants on the surface of the coal. Figure 13 is a scan of the surface of the untreated coal, which shows that the surface has rough, uneven appearance with several crevices. The surface of the coal treated with SDS is depicted in Figure 14. In this micrograph, rod-like structures are distributed in layered patterns on top of the rough surface of the coal. These rods are attributed to adsorbed surfactants and/or surfactant-molybdenum complexes onto the coal surface. Figure 15 shows the coal surface which has been treated with DDAB followed by molybdenum loading. This 
image shows a smoother, more defined coal surface, with pellets randomly distributed on the surface. The composition of the pellets is unknown at this time.

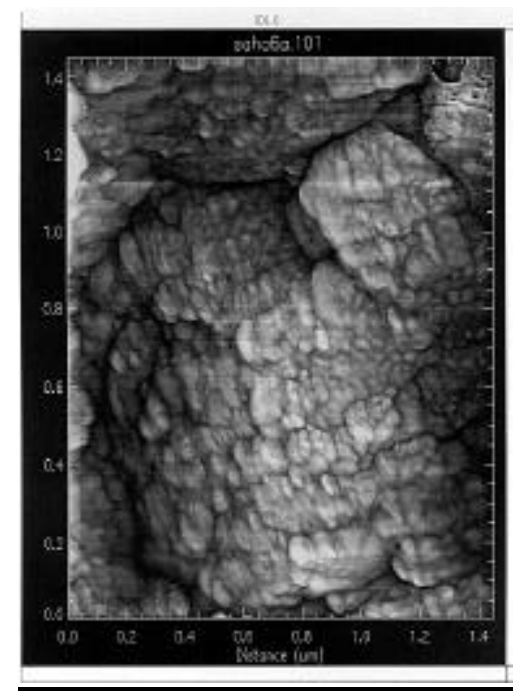

FIGURE 13. Atomic Force Micrograph of Untreated Coal

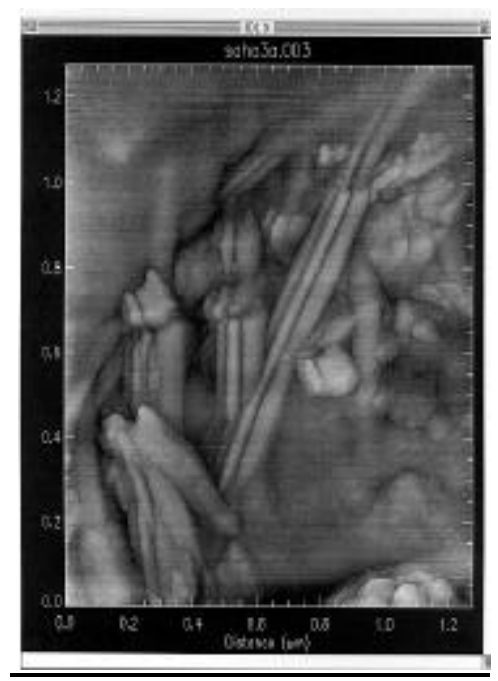

FIGURE 14. Atomic Force Micrograph of the Coal Surface after Treatment with 0.1 M SDS 


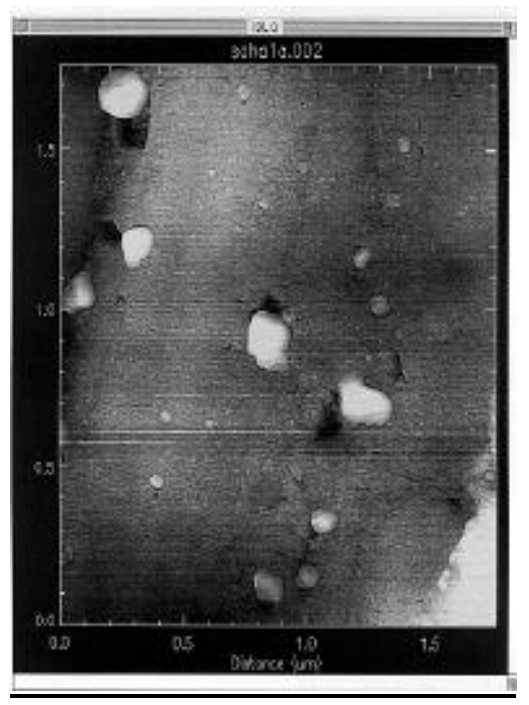

\section{FIGURE 15. Atomic Force Micrograph of Coal Surface after Treatment with 0.1 M DDAB}

\subsection{Luminescence Intensity Measurements}

Luminescence intensity measurements were conducted as a function of sample treatment. The measured luminescence intensity of the unprepared Illinois No. 6 coal was found to be significantly lower $(0.49+/-0.06)$ than was measured a year ago at the Coal and Organic Petrology Labs at Penn State $(0.66+/-0.04)$. This may reflect surface deterioration resulting from extended exposure to air, sunlight or other laboratory conditions, such as oxidation.

Sodium dodecyl sulfate (SDS) appeared as a transparent/translucent material exhibiting crystal structures when observed in reflected white light. Crystals were sometimes separated or intersected by veins of fine-textured granular material. SDS had the lowest measured luminescence intensity of all the specimens evaluated, perhaps because of its transparent nature which may be responsible for internal re-adsorption of the emitted light energy. 
TABLE 6. Mean Luminescence Intensity at $600 \mathrm{~nm}$

\begin{tabular}{|l|l|l|l|}
\hline Sample ID. & $\begin{array}{l}\text { Luminescence } \\
\text { Intensity \% F I600 }\end{array}$ & $\begin{array}{l}\text { Standard } \\
\text { Deviation }\end{array}$ & Confidence $\mathbf{9 5 \%}$ \\
\hline SDS- Surfactant & 0.287 & 0.093 & 0.042 \\
\hline DDAB-Surfactant & 0.512 & 0.282 & 0.129 \\
\hline DECS-24 & 0.487 & 0.125 & 0.056 \\
\hline coal w/ 0.1 M SDS & 0.546 & 0.135 & 0.061 \\
\hline coal w/SDS and Mo & 0.443 & 0.079 & 0.036 \\
\hline coal w/ 0.1 M DDAB & 0.555 & 0.197 & 0.080 \\
\hline coal w/ DDAB + Mo & 0.569 & 0.127 & 0.057 \\
\hline coal w/ 0.1 M Triton & 0.527 & 0.087 & 0.039 \\
\hline coal w/ Triton + Mo & 0.523 & 0.112 & 0.050 \\
\hline
\end{tabular}

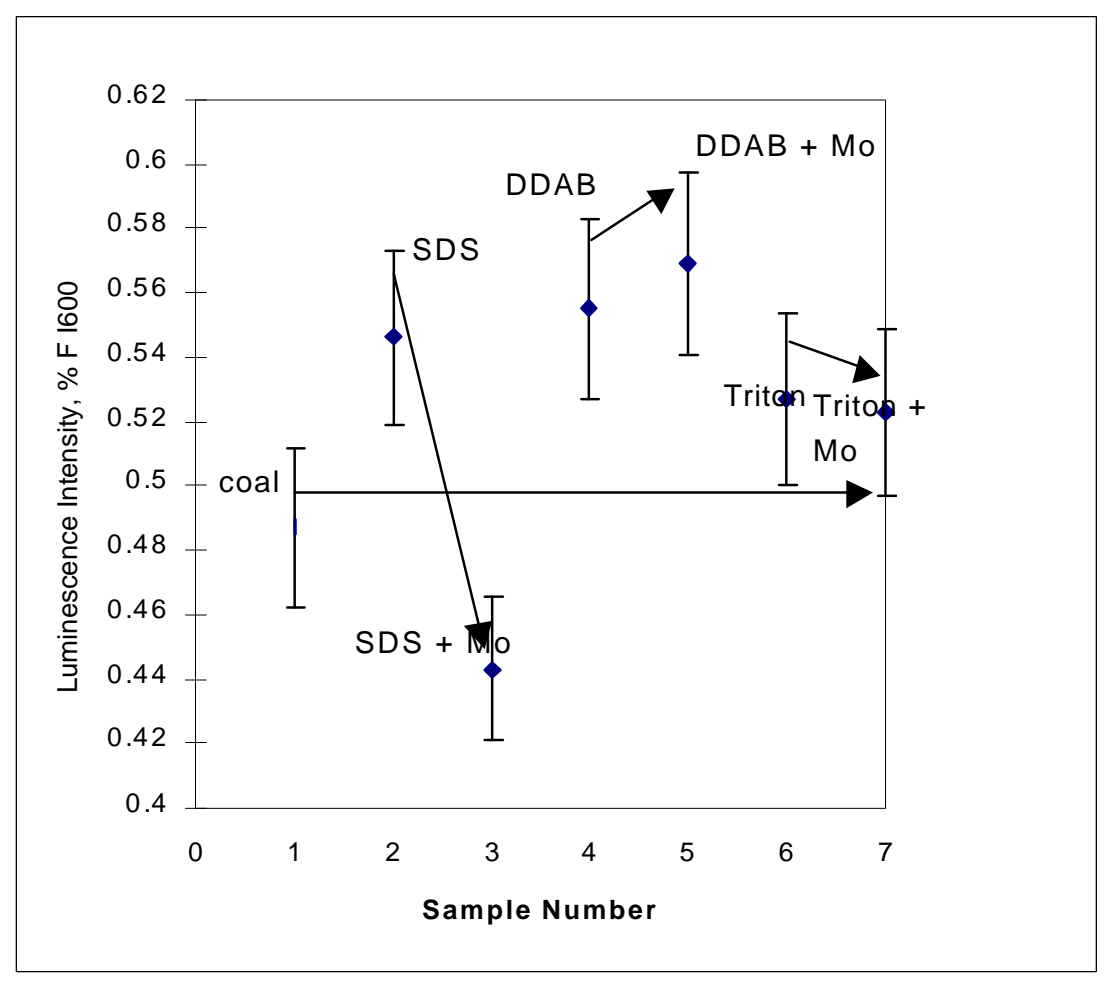

FIGURE 16. Variation in luminescence intensity resulting from the presence of surfactant and molybdenum catalyst on the coal surface 
DDAB appeared to be a wax-like substance that is easily deformed and readily aggregates when attempting to crush. The material exhibits higher luminescence compared to SDS. Under white and blue reflected light, DDAB appeared as formless, rounded particles and aggregates with no crystal edges or cleavage being observed. Luminescence intensity was not measured on the pure Triton X-100 sample.

The influence of surfactants on the luminescence properties of the Illinois No. 6 coal are shown in Table 6 and depicted graphically in Figure 16. There appears to be a slight intensity increase over the mean of the coal when contacted with surfactants. However, as demonstrated in the figure, this increase is not statistically significant.

The SDS surfactant was observed most directly in contact with coal surfaces, but some was seen surrounding coal particles as well as independent crystals not associated with coal particles. Many of the higher intensity readings taken were obtained from coated areas with the coal surface clearly visible through the coating.

On the other hand, the DDAB surfactant commonly was observed as separate particles either not associated with the coal particles or attached at a single point. One very high intensity reading was taken from an irregular depression on a coal surface that is assumed to contain the DDAB surfactant.

Triton X-100 was not identified on the surface. Other than showing a slightly higher mean intensity, no other differences were observed when compared with the untreated coal sample.

Dispersion of the molybdenum catalyst should not have much influence on the luminescence intensity other than obscuring the coal surface and for the most part this is confirmed by the data in Figure 16. This is provided that no carrier solvents were used to dissolve or distribute the catalyst. However, a fairly large decrease in mean intensity was measured for the sample containing SDS and catalyst that can not be explained. Observation of this sample under white and blue light revealed SDS surrounding and coating 
particles in the manner previously described. Presumably, the molybdenum catalyst can be observed in the specimen in two forms: small $(5-10 \mu \mathrm{m})$ clusters of dark iridescent particles that are observed attached sporadically to the coal surface or in direct contact with SDS, and catalyst that is also found as independent clusters $(10-20 \mu \mathrm{m})$ associated with SDS. In no area was a uniform, even coating of catalyst or SDS and catalyst seen associated with coal surfaces.

Inspection of specimens containing Triton and DDAB in association with the molybdenum catalyst did not reveal much of the surfactant. However, in both cases the clusters of catalysts were small $(5-10 \mu \mathrm{m})$ and, qualitatively appeared to be more uniformly distributed on the coal surface. This does not mean that there was an even uniform coating, just that most every coal particle had some associated catalyst.

From the series of samples studied, luminescence intensity measurements show that the coal and solid surfactants luminescence weakly. Also, no statistically significant influence was observed that resulted from the action of the surfactants or surfactantmolybdenum catalyst. Qualitative inspection of the natural fracture surfaces of these treated coals, however, shows that the SDS surfactant may effectively coat coal surfaces and may influence the dispersion of catalyst particles. Catalyst appeared to be better distributed among coal particles and in finer clusters when the DDAB and Triton surfactants were employed.

\subsection{Liquefaction Results}

Measurements of the liquefaction activities produced results that were unexpected based on previously report molybdenum uptake data. Pretreatment of the coal with surfactants produced no significant change in liquefaction activity.

Catalytic activities of surfactant treated coals were lower than the untreated coal sample. This trend was observed in both THF soluble products and heptane soluble products. In 
the absence of a catalyst, $33.8 \%$ heptane solubles was obtained with the parent coal, compared to $27.8 \%$ and $27.3 \%$ with SDS and DDAB respectively. However, the Triton treated sample had a higher conversion of THF soluble products, but similar heptane soluble conversion when compared to the untreated coal sample.

The presence of molybdenum resulted in higher conversion levels of heptane solubles compared with the parent coal. In the absence of surfactants $50 \%$ heptane solubles was obtained, compared to 44-47\% with surfactants. The catalytic activity of molybdenum was more effective on the coal samples treated with SDS and Triton. The coal sample treated with DDAB had the highest amount of molybdenum adsorption, yet the lowest percentage of heptane soluble products.

It is our hypothesis, that the decrease in heptane solubles with surfactants may be due to a blockage of the coal surface from molybdenum due to the coating of the surface with the surfactant. This could inhibit the extent of direct contact between molybdenum and the coal surface and impair catalytic activity.

TABLE 7. Liquefaction activities of untreated and surface modified coal samples.

\begin{tabular}{|l|l|l|}
\hline Sample & $\begin{array}{l}\text { THF Solubles } \\
\text { (Total Coal Conversion to } \\
\text { Liquids) }\end{array}$ & $\begin{array}{l}\text { Heptane } \\
\text { Solubles } \\
\text { (Oils extracted from } \\
\text { THF solubles) }\end{array}$ \\
\hline Original Coal & $72.4 \%$ & $33.8 \%$ \\
\hline Coal + Mo & $90.0 \%$ & $50.0 \%$ \\
\hline Coal + Triton & $77.9 \%$ & $33.2 \%$ \\
\hline Coal +SDS & $70.5 \%$ & $27.8 \%$ \\
\hline Coal + DDAB & $66.6 \%$ & $27.3 \%$ \\
\hline Coal + Triton + Mo & $96.2 \%$ & $46.7 \%$ \\
\hline Coal + SDS + Mo & $95.4 \%$ & $44.5 \%$ \\
\hline Coal + DDAB + Mo & $88.5 \%$ & $46.9 \%$ \\
\hline
\end{tabular}




\subsection{Qualitative analysis of surfactant adsorption by UV measurements}

Based upon the results produced from the liquefaction study, further investigation was needed to reach some conclusion regarding the slight difference in heptane soluble materials of untreated and treated coal samples. The method of surfactant/catalyst loading may have been a factor in the outcome of heptane soluble materials. The concentration of surfactants loaded onto the samples may also have caused unexpected results.

To gain knowledge regarding adsorption at the solid/liquid interface, we established the adsorption isotherm. According to the literature, surfactant molecules bind to active sites at low concentrations horizontal to the surface, due to electrostatic interactions. With an increase in surfactant adsorption, their orientation becomes perpendicular to the surface, at which point the surface coverage increases until all bonding sites are occupied. As surface coverage increases, the surface charge decreases and tends to zero due to ion pairing. After the formation of a monolayer of surfactant, hydrophobic interaction between the tails of the surfactants may begin to form hemmimicelles causing charge reversal. It is at this point that excess surfactant may be expelled into the solution.

With the use of the UV-Vis Spectrophotometer the amount of surfactant adsorbed on the coal surface was measured by testing the difference in the concentration of the surfactant solution loaded and that of the supernatent after loading. As shown by the curve, the amount of surfactant adsorbed steadily increased to a maximum at $147 \mathrm{mg} / \mathrm{g}$ and then decreased. According to Figure 17 the greatest amount of Triton adsorption occurs at approximately $147 \mathrm{mg} / \mathrm{g}$ of coal which corresponds to an equilibrium concentration of $1.6 \mathrm{mmol} / \mathrm{L}$ of solution.

Based upon this experiment, $50 \mathrm{ml}$ of $1.6 \times 10^{-3} \mathrm{M}(1.6 \mathrm{mmol} / \mathrm{L})$ Triton solution is the maximum amount of surfactant solution that can be adsorbed by $0.20 \mathrm{~g}$ of coal to reach maximum saturation. According to the calculations, the amount of surfactant loaded to 2 grams of coal in our initial experiment corresponds to $25 \mathrm{~mL}$ of $2.5 \mathrm{mmol} / \mathrm{L}$ of surfactant 
solution. According to Figure 17, this amount of surfactant solution is shown on the declining side of the isotherm, denoting a loss of surfactant solution. This concentration is nearly five times the critical micelle concentration of Triton $\mathrm{X}-100\left(3.3 \times 10^{-4} \mathrm{M}\right)^{25}$ surfactant. Thus, the surface may have been overloaded with surfactant solution.

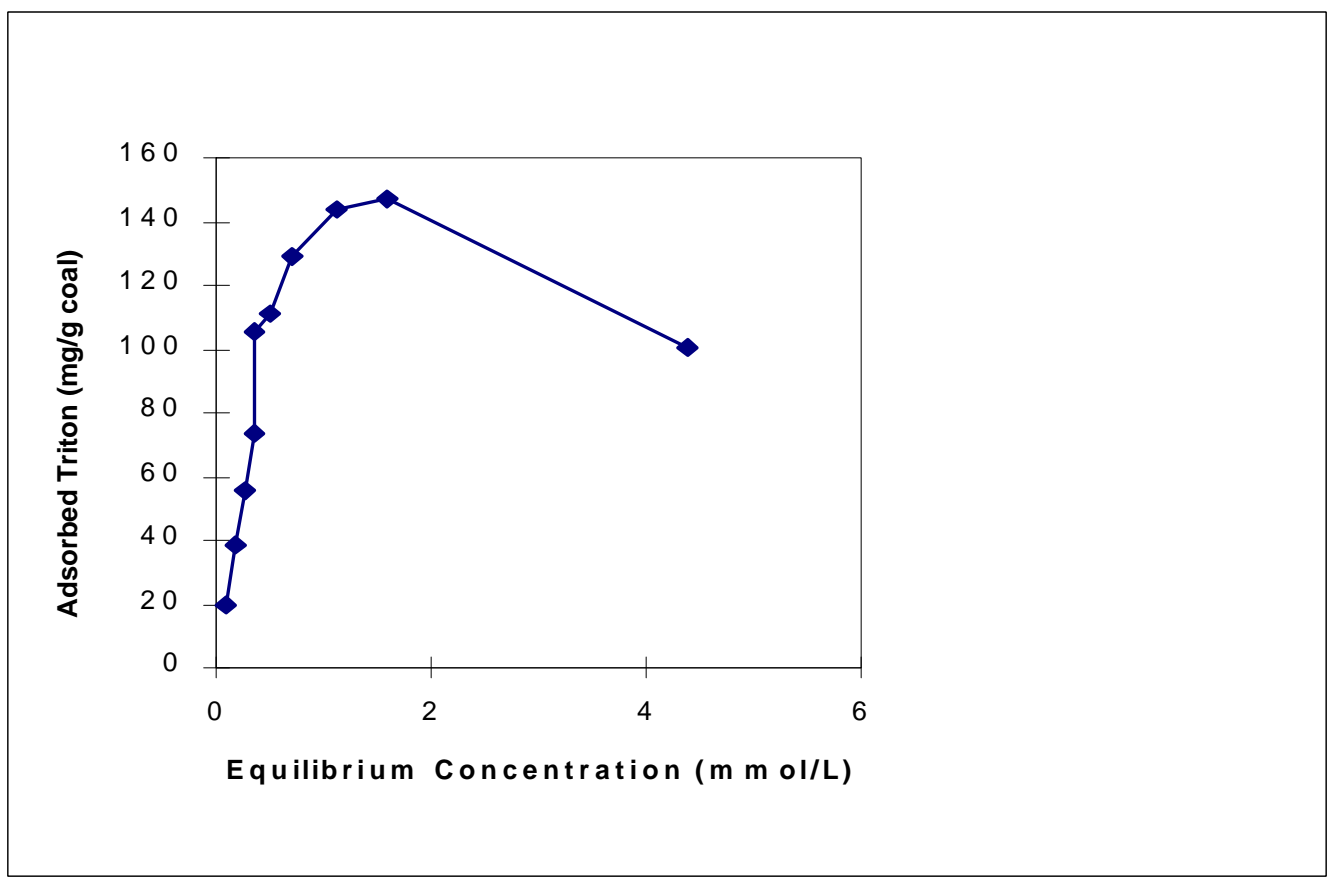

Figure 17. Triton Adsorption Isotherm

\section{OTHER PROGRAM ACCOMPLISHMENTS}

\subsection{Students Supported}

The following students were supported by this project:

- Stacey L. Mayes-Graduated with BS/MS in chemistry, May 1999

- Corey Oliver- Junior in Chemical Engineering

- Allyson Coleman - sophomore in Chemical Engineering

\subsection{Student Thesis Produced}

The following thesis was produced through this project: Surface-modified coals for enhanced catalyst dispersion and liquefaction by Stacey L. Mayes, A thesis for the Degree of Master of Science in Chemistry, May 1999. 


\subsection{Publication and Presentations}

- Mayes, S.; Bota, K.B.; Abotsi, G.; Saha, G., American Chemical Society Preprints of Symposia, 1997, 42 (4), 1058-1061.

\subsection{Special Awards}

- Stacey L. Mayes won the first place award at the annual HBCU student poster research competition in 1997 for her work on this project.

\section{CONCLUSIONS}

- It was shown that the adsorption of molybdenum onto Illinois No. 6 coal (DECS24) is significantly promoted by preadsorption of dodecyl dimethyl ethyl ammonium bromide (DDAB), and sodium dodecyl sulfate (SDS).

- Molybdenum adsorption measurements showed that over a wide range of concentrations and $\mathrm{pH}$ values, the DDAB treated coal adsorbed a higher amount of molybdenum, than the coal sample treated with SDS

- Infrared Spectroscopy results also provided evidence that the untreated coal had the greatest affinity for DDAB.

- Atomic Force Microscopy results were consistent with results observed earlier. The treatment of the coal surface with the surfactants produced obvious differences in the surface of the coal. 
- Luminescence intensity measurements showed that the coal and solid surfactants luminescence weakly. No statistically significant influence was observed that resulted from the action of the surfactants or surfactant-molybdenum catalyst.

- Liquefaction results produced data that indicated the use of surfactants did not significantly improve the liquefaction activity of the coal as had been hypothesized.

- Results obtained from the UV-adsorption experiments provided information regarding the adsorption of the Triton treated coal. It was shown that the surface of the coal was greatly oversaturated. This may have been the cause of the unexpected liquefaction results. However, further experimentation is needed to confirm this hypothesis.

\section{RECOMMENDATIONS}

Further studies regarding factors such as the proper concentration of surfactant and the method of surfactant/catalyst addition would be most useful to enhance such a project. Adsorption isotherms should be generated for each sample of surfactant treated coal. This will provide better accuracy in quantitatively determining the proper amount of surfactant which should be adsorbed to achieve maximum catalyst loading. In addition to this, a more comprehensive study of molybdenum adsorption as a result of Triton X-100 preadsorption onto coal should provide a great amount of useful information.

In addition, comparisons of different catalysts such as iron and calcium may provide more information regarding the results produced in this experiment. 


\section{ACKNOWLEDGMENTS}

This project was proposed and initiated by Dr. Godfried K. Abotsi who suddenly passed away in1997. The entire outcome of the project is to his credit. May he rest in peace.

Special thanks go to:

- Ms. Stacey L. Mayes, Clark Atlanta University, for her outstanding work in this project.

- Dr. Anthony Cugini, Federal Energy Technology Center, Pittsburgh, PA.; Dr. Paul Bertsch, Doug Hunter, and Emmanuel Montarges of SREL- University of Georgia; and Dr. Gareth Mitchell, Coal and Organic Petrology Laboratory of Pennsylvania State University for their scientific contributions which made the completion of this project possible.

- Dr. Yong Xu for his contribution in the preparation of this report.

- U.S. Department of Energy under grant number DE-FG22-95PC95229 for the Financial support for this project.

10 REFERENCES

1. Ward, Colin, Ed.; Coal Geology and Technology ; Blackwell Scientific: Boston, MA., 1984 ; pp 1-33.

2. Blaustein, B., Suhy, D., Spana, E., Am. Chem. Soc., Div. Fuel Chem. Prepr., 1990, 35(3), 919.

3. Donath, E.E.; Hoering, M. Fuel Proc. Tech. 1977, 1, 3.

4. Tanabe, K., Hattori, H., Yamaguchi, T., Lizuka, T., Matsuhashi, H., Kimura, A., Nagase, Y., Fuel Proc. Tech. 1986, 14,247.

5. Hatswell, M.R.; Jackson, W.r.; Larkins, F.P.; Marshall, M.; Rash, D.; Rogers, D. E., Fuel 1983, 62, 336.

6. Cillo, D.L.; Stiegel, G. J.; Tishcher, R.E.; Narain, N.K., Fuel Proc. Tech. 1986, 13,65 . 
7. Tishcer, R.E.; Narain, N.K.; Stiegel, G.J.; Cillo, D.L. Journal of Catalysis 1985, 95, 406.

8. Ternan, M.; Brown, J.R., Fuel 1982, 61, 1110.

9. "Report on the Petroleum and Synthetic Oil Industry of Germany, “Ministry of Fuel and Power (U.K.) 1947.

10. Lytle, J.M.; Hsieh, B.C.; Anderson, L.L.; Wood R.E., Fuel Proc. Tech. 1979, 235.

11. Wurfel, H.E., Fuel Proc. Tech. 1979, 2, 227.

12. Rao, A.K.; Gadiyar, H.J.; Pate, F.L. "Catalytic Hydrogenation of SRC-1 Product at the Wilsonville Pilot Plant." 7th EPRI Contractors Conference, Palo Alto, CA 1982.

13. Schindler, H.D.; Chen J.M.; Peluso, M.; Mnoroni, E.C.; Potts, J.D. “The Integrated Two Stage Liquefaction Process (ITSL), “ AIChE Meeting, New Orleans, LA 1981.

14. Cugini, A. V.; Lett, R.G., Energy and Fuels 1989, 3 (2), 120.

15. Bockrath, B.; Illig, E., Keller, M.; Hough, M., Direct Liquefaction Contractors Review Meeting, Pittsburgh, PA 1989, 201.

16. Cugini, A. V.; Utz, B.R.; Krastman, D.; Lett, R. G.;Balsone, V., Proceedings of 15th Int. Conf. Coal \& Slurry Technology, Clearwater, FL. 1990, 331.

17. Farcasiu, M.; Smith, C., Energy and Fuels 1989, 3, 562.

18. Utz, B.R.; Cugini, A.V.; Frommell, E.A., American Chemical Society, Divsion of Fuel Chemistry Preprints 1989, 34 (4), 1423.

19. Schobert, H.H. "Coal: The Energy Source of the Past and Future." American Chemical Society, Washington, D.C., 1987.

20. Fuerstenau, D.W., Ed.; Froth Flotation 50th Anniverssry Volume, The American Institiute of Mining, Mettallurgical, and Petroleum Engineers: New York, N.Y.; pp. 518-537.

21. Abotsi, G.K. , Bota Kofi, Saha G.; Energy and Fuels 1992, 6, 779-782.

22. Mochida, I., Sakanishi, Catalysis in Coal Liquefaction, Academic Press: New York, N.Y.; pp 39-85. 
23. Botsaris, G., and Yuli, M., Interfacial Phenomena in Coal Technology, Marcel Dekker: 1989.

24. Botsaris, G., and Yuli, M., Interfacial Phenomena in Coal Technology, Marcel Dekker: 1989.

25. Yamada, O.; Suzuki, T., Then, J.H., Ando. T., Watanabe, Y., Fuel Proc. Tech. 1985, 11, 297.

26 Kamiya, Y.; Obusawa, T., Futamura, S., Fuel Proc. Tech. 1988, 18, 1.

27. Fuerstenau, D.W.; Pradip, Colloids and Surfaces 1982, 4, 229-243.

28. Stranges, A., Fuel Proc. Tech. 1987, 16, 205-225.

29. Wurfel, H., Fuel Proc. Tech. 1979, 2, 227-233.

30 Watanabe, Y.;Yamada, O., Fujita, K.; Suzuiki, T., Fuel 1984, 63, 752.

31. Suzuki, T.; Yamada, O.; Fujita, K., Takegami, Y., Watanabe, Y., Fuel 1984, 63, 1706.

32. Garg, D.; Givens, E.N., Ind. Eng. Chem. Process Des. Dev. 1982, 21, 113.

33. Garg, D.; Givens, E.N, Fuel Proc. Tech. 1983, 7, 59.

34. Huang, W.J.; Curtis, C.W.; J.A., Clinton, J.H., Tarrer, A.R., AIChE Preprint 1981, 93.

35. Brooks, D.G.; Guin J.A.; Curtis, C.W., Placet, T.D., Ind.Eng. Chem. Process Des. Dev. 1983, 22, 43.

36. Pradhan, V.R.; Herrick, D.E.; Teirney, J.W., Wender, I., Energy \& Fuels 1991, 5,712 .

37. Warzinski, R.P.; Lee, C.; Holder, G.D., Journal of Supercritical Fluids 1992, 5, 60.

38. Andres, M.; Charcosset, H.; Chiche, P., Davignon, L., Fuel 1983, 62, 690.

39. Schlesinger, M.D.; Frank, L.V.; Hiteshue, R., Bureau of Mines Report on Investigations No. 6021, 1962.

40. Weller, S.W.; Pelipetz, M. G., Ind. Eng. Chem. 1951, 43, 1243.

41. Yamashita, H.; Ohtsuka, Y.; Yoshida, S.; Tomita, A., Energy \& Fuels 1989, 3, 686.

42. Vittal, M.; Boehman, A., Energy \& Fuels 1996, 10, 1028-1029.

43. Schobert, H.H. "Coal: The Energy Source of the Past and Future." 
American Chemical Society, Washington, D.C., 1987.

44. Johnson, J.L. In "Fundamental fo Coal Utilization: 2nd Supplementary

Volume, " Elliott, M.A., Ed.; John Wiley: New York, 1981.

45. Suzuki, T.; Yamada, O.; Takahashi, Y., Watanabe, Y., Fuel Proc. Tech. $1985,10,33$

46. Yamada, O.; Suzuki, T., Then, J. H., Ando, T., Waranabe, Y., Fuel Proc. Tech. 1985, 11, 297.

47. Kamiya, Y.; Obusawa, T., Futamura, S., Fuel Proc. Tech. 1988, 18,1.

48. Guin, J.A.; Tarrer, A.R.; Prather, J.W., Johnson, D.R. Lee, J.M., Ind. Eng. Chem. Process Des. Dev., 1978, 17, 118.

49. Mukhejee, D.K.; Chowdhury, P.B., Fuel 1976, 55, 4.

50. Huang, W.J.; Curtis, C. W.; Guin, J.A., Clinton, J.H., H.L., Tarrer, A.R., AIChE Preprints 1981, 93.

51. Mazzocco, N.J.; Klunder, E.B.; Krastman, D., United State Department of Energy Report No. DOE/PETC/TR-81/1, 1981.

52. Brooks, D.G.; Guin, J.A.; Curtis, C.W., Placet, T.D., Ind. Eng. Chem. Process Des. Dev. 1983, 22, 43.

53. Pradhan, V.R.; Herrick, D.E.; Tierney, J.W., Wender, I., Energy \& Fuels $1991,5,712$.

54. Pradhan, V.R., Tiemey, J.W., Wender, I., Energy \& Fuels 1991, 5, 497.

55. Osipaw, L., Surface Chemistry, Reinhold:New York, N.Y., 1990.

56. Brown, D. J., In Froth Flotation; 50th Anniversary AIME: New York, 1962.

57. Cassassa, E.Z.; Parfit. G.D.; Rao, A.S.; Toor, E.W.. "Proceedings 6th Int. Symposium on Coal and Slurry Combustion and Technology. " Pittsburgh, PA 1984, p 251.

58. Fuerstenau, D.W.; Somasundaran, P., Journal of Physical Chemistry 1966, 70, 90-96.

59. Mayes, S.; Bota, K.B.; Abotsi, G.; Saha, G., American Chemical Society Prepints of Symposia, 1997, 42 (4), 1058-1061.

60 Richardson, J.T., Principles of Catalyst Development, Plenium Press:New York, 1989, pp 146-151. 